\title{
INEQUALITIES AND BOUNDS FOR THE EIGENVALUES OF THE SUB-LAPLACIAN ON A STRICTLY PSEUDOCONVEX CR MANIFOLD
}

\author{
AMINE ARIBI AND AHMAD EL SOUFI
}

\begin{abstract}
Aвstract. We establish inequalities for the eigenvalues of the sub-Laplace operator associated with a pseudo-Hermitian structure on a strictly pseudoconvex CR manifold. Our inequalities extend those obtained by Niu and Zhang [26] for the Dirichlet eigenvalues of the sub-Laplacian on a bounded domain in the Heisenberg group and are in the spirit of the well known Payne-Pólya-Weinberger and Yang universal inequalities.
\end{abstract}

\section{INTRODUCTION}

The sub-Laplacian $\Delta_{b}$ associated with a pseudo-Hermitian structure on a strictly pseudoconvex CR manifold $M$ is prototypical of a class of subelliptic operators which appear naturally in several geometric situations that could be gathered under the concept of "Heisenberg manifolds". The recent work of Ponge [29] provides a detailed discussion and a fairly comprehensive presentation of the spectral properties of such operators, including Weyl asymptotic formulae and heat kernel expansions.

The sub-Laplacian $\Delta_{b}$ plays a fundamental role in CR geometry, similar to that played by the Laplace-Beltrami operator in Riemannian geometry (e.g., CR Yamabe problem). Several works published in recent years are devoted to the study of this operator and the investigation of its spectral properties, see for instance [3, 4, 5, 8, 17, 24, 26, 29]. In particular, it is known that $\Delta_{b}$ is subelliptic of order $\frac{1}{2}$, that is for each $x \in M$, there exist a neighborhood $U \subset M$ and a constant $C>0$ such that, $\forall u \in C_{0}^{\infty}(U)$,

$$
\|u\|_{H^{1 / 2}}^{2} \leq C\left\langle\left(-\Delta_{b}+I\right) u, u\right\rangle_{L^{2}}
$$

This a priori estimate leads to the proof of the hypoellipticity of $\Delta_{b}$ and the discreteness of its spectrum when $M$ is a closed manifold (see [3, 5, 25]). Since the pioneering work of Greenleaf [17], many recent contributions aim to extend to the CR context some of the spectral geometric results established in the Riemannian setting such as Li-Yau or Lichnerowicz-Obata inequalities (see, for example, [3, 4, 8, 24]). It is worth noticing that the determination of the eigenvalues of the sub-Laplacian on the standard CR

2000 Mathematics Subject Classification. 32V20, 35H20, 58J50.

Key words and phrases. CR manifold, sub-Laplacian, eigenvalue, Carnot group.

The second author has benefited from the support of the ANR (Agence Nationale de la Recherche) through FOG project ANR-07-BLAN-0251-01. 
sphere $\mathbb{S}^{2 n+1}$ remains an open problem, except likely for $n=1$ according to [28].

In this article, we focus on finding bounds on the eigenvalues in the same vein as Payne-Pólya-Weinberger universal inequalities [27]. These inequalities, established in the 1950's for the eigenvalues of the Dirichlet Laplacian in a bounded domain of the Euclidean space $\mathbb{R}^{n}$, were first stated as follows: for every $k \geq 1$,

$$
\lambda_{k+1}-\lambda_{k} \leq \frac{4}{n}\left\{\frac{1}{k} \sum_{i=1}^{k} \lambda_{i}\right\}
$$

before being improved by several authors (see for instance [1, 23, 30]). For example, the following inequality due to Yang [30] implies (1.1) :

$$
\sum_{i=1}^{k}\left(\lambda_{k+1}-\lambda_{i}\right)^{2} \leq \frac{4}{n} \sum_{i=1}^{k} \lambda_{i}\left(\lambda_{k+1}-\lambda_{i}\right) .
$$

Extensions of universal inequalities to bounded domains in Riemannian manifolds other than the Euclidean space have also been obtained. Let us mention, for example, the following Yang's type inequality obtained by Ashbaugh [1] for domains of the unit sphere $\mathbb{S}^{n} \subset \mathbb{R}^{n+1}$ (see also [10]):

$$
\sum_{i=1}^{k}\left(\lambda_{k+1}-\lambda_{i}\right)^{2} \leq \frac{4}{n} \sum_{i=1}^{k}\left(\lambda_{k+1}-\lambda_{i}\right)\left(\lambda_{i}+\frac{n^{2}}{4}\right) .
$$

It is a remarkable fact that the equality holds for every $k$ in this last inequality when the $\lambda_{i}$ are the eigenvalues of the Laplace-Beltrami operator on the whole sphere. This fact was observed by El Soufi, Harrell and Ilias in their paper [16] where inequality (1.3), as well as many other inequalities in the literature, are recovered as particular cases of the following inequality which applies to the eigenvalues of the Laplace-Beltrami operator of any $n$-dimensional compact Riemannian manifold $M$, with Dirichlet boundary conditions if $\partial M \neq \emptyset$,

$$
\sum_{i=1}^{k}\left(\lambda_{k+1}-\lambda_{i}\right)^{2} \leq \frac{4}{n} \sum_{i=1}^{k}\left(\lambda_{k+1}-\lambda_{i}\right)\left(\lambda_{i}+\frac{1}{4}\|H\|_{\infty}^{2}\right),
$$

where $H$ is the mean curvature vector field of any isometric immersion of $M$ into a Euclidean space $\mathbb{R}^{n+p}$. Notice that inequality (1.4) had also been found independently by Chen and Cheng [9] for the Dirichlet eigenvalues on a bounded domain of a Riemannian manifold.

Niu and Zhang [26] were certainly the first to address this issue for subelliptic operators. They obtained Payne-Pólya-Weinberger and Hile-Protter type inequalities for the Dirichlet eigenvalues of the sub-Laplacian on a bounded domain of the Heisenberg group $\mathbb{H} \mathbb{H}^{n}$ of real dimension $2 n+1$. The following Yang type inequality has been obtained in this context in [16] as 
an improvement of Niu-Zhang results:

$$
\sum_{i=1}^{k}\left(\lambda_{k+1}-\lambda_{i}\right)^{2} \leq \frac{2}{n} \sum_{i=1}^{k} \lambda_{i}\left(\lambda_{k+1}-\lambda_{i}\right) .
$$

In what follows (see Corollary 4.1 below), we will prove that inequality (1.5) remains valid for any strictly pseudoconvex CR manifold $M$ of real dimension $2 n+1$ provided it admits a Riemannian submersion over an open set of $\mathbb{R}^{2 n}$ which is constant along the characteristic curves of $M$ (i.e. the integral curves of the Reeb vector field). Of course, the standard projection $\mathbb{H} \mathbb{H}^{n} \rightarrow \mathbb{R}^{2 n}$ satisfies these assumptions.

As for the CR sphere $\mathbb{S}^{2 n+1}$ and domains of $\mathbb{S}^{2 n+1}$, we will obtain the following inequality (Corollary 3.1):

$$
\sum_{i=1}^{k}\left(\lambda_{k+1}-\lambda_{i}\right)^{2} \leq \frac{2}{n} \sum_{i=1}^{k}\left(\lambda_{k+1}-\lambda_{i}\right)\left(\lambda_{i}+n^{2}\right),
$$

which is sharp for $k=1$.

All these results are actually particular cases of a more general result (Theorem 3.1) that we establish in Section 3 for an arbitrary strictly pseudoconvex CR manifold $M$ of real dimension $2 n+1$ endowed with a compatible pseudo-Hermitian structure $\theta$. Indeed, we prove that the eigenvalues of the sub-Laplacian $\Delta_{b}$ in a bounded domain $\Omega \subset M$, with Dirichlet boundary conditions if $\Omega \neq M$, satisfy inequalities of the form (see Theorem 3.1 for a complete statement): for every integer $k \geq 1$ and every $p \in \mathbb{R}$,

$$
\begin{gathered}
\sum_{i=1}^{k}\left(\lambda_{k+1}-\lambda_{i}\right)^{p} \leq \frac{\max \{2, p\}}{n} \sum_{i=1}^{k}\left(\lambda_{k+1}-\lambda_{i}\right)^{p-1}\left(\lambda_{i}+\frac{1}{4}\left\|H_{b}(f)\right\|_{\infty}^{2}\right), \\
\lambda_{k+1} \leq\left(1+\frac{2}{n}\right) \frac{1}{k} \sum_{i=1}^{k} \lambda_{i}+\frac{1}{2 n}\left\|H_{b}(f)\right\|_{\infty}^{2},
\end{gathered}
$$

and

$$
\lambda_{k+1} \leq\left(1+\frac{2}{n}\right) k^{\frac{1}{n}} \lambda_{1}+\frac{1}{4}\left(\left(1+\frac{2}{n}\right) k^{\frac{1}{n}}-1\right)\left\|H_{b}(f)\right\|_{\infty}^{2},
$$

where $f$ is any $C^{2}$ semi-isometric map from $(M, \theta)$ to a Euclidean space $\mathbb{R}^{m}$, and where $H_{b}(f)$ is a vector field defined similarly to the tension vector field in the Riemannian case (see Section 2 for definitions).

Besides the CR sphere and Heisenberg groups, many other cases in which one has an explicit expression for $\left\|H_{b}(f)\right\|_{\infty}$ are given in a series of corollaries in Section 4.

In Section 5 we prove that the inequalities (1.6), (1.7) and (1.8) remain true when $f$ is a semi-isometric map from $(M, \theta)$ to a Heisenberg group $\mathbb{H} \mathbb{H}^{m}$ which sends the horizontal distribution of $M$ into that of $\mathbb{H}^{m}$. This can also be seen as a generalization of what was known about the Dirichlet eigenvalues of the sub-Laplacian in a bounded domain of the Heisenberg group, since the identity map of $\mathbb{H}^{n}$ obviously satisfies $H_{b}\left(I_{\mathbb{H}^{n}}\right)=0$. 
When $M$ is compact without boundary, one has $\lambda_{1}=0$ and the inequality (1.8) leads to a relationship between the eigenvalues $\lambda_{k}$ of the sub-Laplacian of $(M, \theta)$ and the invariant $H_{b}(f)$ of any semi-isometric map $f$ from $(M, \theta)$ to a Euclidean space. For the first positive eigenvalue $\lambda_{2}$, we even have the following inequality :

$$
\lambda_{2}\left(-\Delta_{b}\right) \leq \frac{1}{2 n V(M, \theta)} \int_{M}\left|H_{b}(f)\right|_{\mathbb{R}^{m}}^{2},
$$

where $V(M, \theta)$ is the volume of $(M, \theta)$. Section 6 deals with these Reilly type inequalities and the characterization of equality cases. For example, we show that the equality holds in (1.9) if and only if $f(M)$ is contained in a sphere $\mathbb{S}^{m-1}(r)$ of radius $r=\sqrt{\frac{2 n}{\lambda_{2}\left(-\Delta_{b}\right)}}$ and $f$ is a pseudo-harmonic map from $M$ to $S^{m-1}(r)$.

These Reilly type results are also extended to maps $f$ from $(M, \theta)$ to a Heisenberg group $\mathbb{H}^{m}$ which sends the horizontal distribution of $M$ into that of $\mathbb{H}^{m}$ (see Theorem 6.2).

The last part of the paper deals with Carnot groups which constitute a natural generalization of Heisenberg groups. A Carnot group is equipped with a natural operator called "horizontal Laplacian". We give PPW and Yang type inequalities for the eigenvalues of the horizontal Laplacian in terms of the rank of the horizontal distribution of the group.

Acknowledgments. The authors would like to warmly thank S. Dragomir, N. Gamara, R. Petit and A. Zeghib for useful discussions.

\section{Preliminaries}

Let $M$ be an orientable CR manifold of CR dimension $n$. This means that $M$ is an orientable manifold of real dimension $2 n+1$ equipped with a pair $(H(M), J)$, where $H(M)$ is a subbundle of the tangent bundle $T M$ of real rank $2 n$ (often called Levi distribution) and $J$ is an integrable complex structure on $H(M)$. The integrability condition for $J$ means that, $\forall X, Y \in$ $\Gamma(H(M))$,

$$
[X, Y]-[J X, J Y] \in \Gamma(H(M))
$$

and

$$
[J X, Y]+[X, J Y]=J([X, Y]-[J X, J Y]) .
$$

Since $M$ is orientable, there exists a nonzero 1-form $\theta \in \Gamma\left(T^{*} M\right)$ such that $\operatorname{Ker} \theta=H(M)$. Such a 1-form, called pseudo-Hermitian structure on $M$, is of course not unique. Actually, the set of pseudo-Hermitian structures that are compatible with the CR-structure of $M$ consists in all the forms $f \theta$ where $f$ is a smooth nowhere zero function on $\mathrm{M}$.

To each pseudo-Hermitian structure $\theta$ we associate its Levi form $G_{\theta}$ defined on $H(M)$ by

$$
G_{\theta}(X, Y)=-d \theta(J X, Y)=\theta([J X, Y])
$$


(note that a factor $\frac{1}{2}$ is sometimes put before $d \theta$ so that in the case of the sphere $\mathbb{S}^{2 n+1} \subset \mathbb{C}^{n+1}$, the Webster metric defined below coincides with the standard metric).

The integrability of $J$ implies that $G_{\theta}$ is symmetric and $J$-invariant. The $C R$ manifold $M$ is said to be strictly pseudoconvex if the Levi form $G_{\theta}$ of a compatible pseudo-Hermitian structure $\theta$ is either positive definite or negative definite. Of course, this condition does not depend on the choice of $\theta$. It implies that the distribution $H(M)$ is far from being integrable.

In all the sequel, a pair $(M, \theta)$ will be called strictly pseudoconvex $\mathrm{CR}$ manifold if $M$ is a strictly pseudoconvex CR manifold endowed with a compatible pseudo-Hermitian structure $\theta$ with positive definite Levi form. The structure $\theta$ is then a contact form which induces on $M$ the following volume form

$$
\vartheta_{\theta}=\frac{1}{2^{n} n !} \theta \wedge(d \theta)^{n} .
$$

We will denote by $V(M, \theta)$ the volume of $M$ with respect to $\vartheta_{\theta}$.

A pseudo-Hermitian structure $\theta$ on a strictly pseudoconvex CR manifold determines a vector field $\xi$, often called characteristic direction or Reeb vector field of $\theta$, defined to be the unique tangent vector field on $M$ satisfying $\theta(\xi)=1$ and $\xi\rfloor d \theta=0$. Therefore, $L_{\xi} \theta=0$ and $[H(M), \xi] \subset H(M)$.

The Tanaka-Webster connection of a strictly pseudoconvex CR manifold $(M, \theta)$ is the unique affine connection $\nabla$ on $T M$ satisfying the following conditions :

(1) $\nabla \theta=0, \nabla d \theta=0$ and $\nabla J=0$ (hence the distribution $H(M)$ and the vector field $\xi$ are parallel for $\nabla$ )

(2) The Torsion $T_{\nabla}$ of $\nabla$ is such that, $\forall X, Y \in H(M)$,

$$
T_{\nabla}(X, Y)=-\theta([X, Y]) \xi \quad \text { and } \quad T_{\nabla}(\xi, J X)=-J T_{\nabla}(\xi, X) \in H(M) .
$$

Basic examples : Standard models for $\mathrm{CR}$ manifolds are given by the Heisenberg group and real hypersurfaces of complex manifolds. The Heisenberg group will be discussed in Section 5. If $M$ is an orientable real hypersurface of $\mathbb{C}^{n+1}$, then the sub-bundle $H(M)$ defined as the orthogonal complement of $J v$ in $T M$, where $v$ is a unit normal vector field and $J$ is the standard complex structure of $\mathbb{C}^{n+1}$, is stable by $J$. The pair $(H(M), J)$ endows $M$ with a CR-structure whose compatible pseudo-Hermitian structures are represented by

$$
\theta(X)=-\frac{1}{2}\langle X, J v\rangle,
$$

where $\langle$,$\rangle is the standard inner product in \mathbb{C}^{n+1}$. A straightforward calculation gives

$$
G_{\theta}(X, X)=\frac{1}{2}(B(X, X)+B(J X, J X)),
$$

where $B$ is the second fundamental form of the hypersurface. Thus, $M$ is strictly pseudoconvex if and only if the $J$-invariant part of its second fundamental form is positive definite on $H(M)$. 
Since the second fundamental form of the sphere $\mathbb{S}^{2 n+1} \subset \mathbb{C}^{n+1}$ coincides with the standard inner product, the above construction endows $\mathbb{S}^{2 n+1}$ with a strictly pseudoconvex CR structure whose Levi form is nothing but the restriction of the standard inner product to the horizontal bundle $H\left(\mathbb{S}^{2 n+1}\right)$ where, for every $x \in \mathbb{S}^{2 n+1}, H_{x}\left(\mathbb{S}^{2 n+1}\right)$ is the orthogonal complement in $\mathbb{C}^{n+1}$ of the complex line passing through $x$.

Sub-Laplacian : A Strictly pseudoconvex CR manifold $(M, \theta)$ is equipped with a natural second order differential operator $\Delta_{b}$ commonly known as the "sub-Laplacian". This operator is defined in terms of the Tanaka-Webster connection $\nabla$ by:

$$
\Delta_{b} u=\operatorname{trace}_{G_{\theta}} \nabla d u .
$$

Given a local $G_{\theta}$-orthonormal frame $\left\{X_{1}, \ldots, X_{2 n}\right\}$ of $H(M)$, one has

$$
\Delta_{b} u=\sum_{i=1}^{2 n}\left\{X_{i} \cdot X_{i} \cdot u-\left(\nabla_{X_{i}} X_{i}\right) \cdot u\right\}=\sum_{i=1}^{2 n}\left\langle\nabla_{X_{i}} \nabla^{H} u, X_{i}\right\rangle_{G_{\theta}},
$$

where $\nabla^{H} u \in H(M)$ is the horizontal gradient of $u$ defined by, $\forall X \in H(M)$, $X \cdot u=G_{\theta}\left(X, \nabla^{H} u\right)$. Integration by parts yields for every compactly supported smooth function $u$ on $M$,

$$
\int_{M} u \Delta_{b} u \vartheta_{\theta}=-\int_{M}\left|\nabla^{H} u\right|_{G_{\theta}}^{2} \vartheta_{\theta} .
$$

When $(M, \theta)$ is strictly pseudoconvex, the Levi form $G_{\theta}$ extends to a Riemannian metric $g_{\theta}$ on $M$, sometimes called the Webster metric, so that the decomposition $T M=H(M) \oplus \mathbb{R} \xi$ is orthogonal and the vector $\xi$ has unit length, that is, $\forall X, Y \in T M$,

$$
g_{\theta}(X, Y)=G_{\theta}\left(X^{H}, Y^{H}\right)+\theta(X) \theta(Y),
$$

where $X^{H}=\pi_{H} X$ is the projection of $X$ on $H(M)$ with respect to the decomposition $T M=H(M) \oplus \mathbb{R} \xi$. Notice that the Riemannian volume form associated to $g_{\theta}$ coincides with $\vartheta_{\theta}$ (see [7, Lemma 1]). On the other hand, the Levi-Civita connection $\nabla^{g_{\theta}}$ of $\left(M, g_{\theta}\right)$ is related to the Tanaka-Webster connection $\nabla$ by the following identities (see for instance [13, p.38]): for every pair $X, Y$ of horizontal vector fields, $\nabla_{X} Y=\left(\nabla_{X}^{g_{\theta}} Y\right)^{H}$ and, moreover,

$$
\begin{gathered}
\nabla_{\xi}^{g_{\theta}} X-\nabla_{\xi} X=\frac{1}{2} J X, \quad \nabla_{X}^{g_{\theta}} \xi-\nabla_{X} \xi=\nabla_{X}^{g_{\theta}} \xi=\left(\frac{1}{2} J+\tau\right) X, \\
\nabla_{X}^{g_{\theta}} Y-\nabla_{X} Y=-\left\langle\left(\frac{1}{2} J+\tau\right) X, Y\right\rangle_{g_{\theta}} \xi \quad \text { and } \quad \nabla_{\xi}^{g_{\theta}} \xi=\nabla_{\xi} \xi=0,
\end{gathered}
$$

where $\tau: H(M) \longrightarrow H(M)$ is the traceless symmetric $(1,1)$-tensor defined by $\tau X=T_{\nabla}(\xi, X)=\nabla_{\xi} X-[\xi, X]$. Notice that $\tau=0$ if and only if $\xi$ is a Killing vector field w.r.t. the metric $g_{\theta}$ (and then the metric $g_{\theta}$ is a Sasakian metric on $M$ ). 
If we denote by $\operatorname{div}_{g_{\theta}}$ the divergence with respect to the metric $g_{\theta}$, one easily gets

$$
\Delta_{b} u=\operatorname{div}_{g_{\theta}} \nabla^{H} u,
$$

which immediately leads to the following relationship, known as Greenleaf's formula:

$$
\Delta_{b}=\Delta_{g_{\theta}}-\xi^{2}
$$

where $\Delta_{g_{\theta}}$ is the Laplace-Beltrami operator of $\left(M, g_{\theta}\right)$.

Levi tension vector field : Let $(M, \theta)$ be a strictly pseudoconvex CR manifold of dimension $2 n+1$ and let $(N, h)$ be a Riemannian manifold. The energy density of a smooth $f:(M, \theta) \longrightarrow(N, h)$ with respect to horizontal directions is defined at a point $x \in M$ by

$$
e_{b}(f)_{x}=\frac{1}{2} \operatorname{trace}_{G_{\theta}}\left(\pi_{H} f^{*} h\right)_{x}=\frac{1}{2} \sum_{i=1}^{2 n}\left|d f\left(X_{i}\right)\right|_{h}^{2},
$$

where $\left\{X_{1}, \ldots, X_{2 n}\right\}$ is a local $G_{\theta}$-orthonormal frame of $H(M)$. According to [6. Theorem 3.1], the first variation of the energy functional

$$
E_{b}(f)=\int_{M} e_{b}(f) \vartheta_{\theta}
$$

is determined by the vector, that we will call "Levi tension" of $f$,

$$
H_{b}(f)=\operatorname{trace}_{G_{\theta}} \beta_{f},
$$

where $\beta_{f}$ is the vector valued 2-form on $H(M)$ given by

$$
\beta_{f}(X, Y)=\nabla_{X}^{f} d f(Y)-d f\left(\nabla_{X} Y\right),
$$

$\nabla^{f}$ is the connection induced on the bundle $f^{-1} T N$ by the Levi-Civita connection of $(N, h)$, and $\nabla$ is the Tanaka-Webster connection of $(M, \theta)$. That is,

$$
H_{b}(f)=\sum_{i=1}^{2 n} \nabla_{X_{i}}^{f} d f\left(X_{i}\right)-d f\left(\nabla_{X_{i}} X_{i}\right) .
$$

Mappings with $H_{b}(f)=0$ are called pseudo-harmonic by Barletta, Dragomir and Urakawa [6]. In the case where $(N, h)$ is the standard $\mathbb{R}^{m}$, it is clear that

$$
H_{b}(f)=\left(\Delta_{b} f_{1}, \ldots, \Delta_{b} f_{m}\right) .
$$

Since $\nabla_{X} Y=\left(\nabla_{X}^{g_{\theta}} Y\right)^{H}=\nabla_{X}^{g_{\theta}} Y-\left\langle\left(\frac{1}{2} J+\tau\right) X, Y\right\rangle_{G_{\theta}} \xi$ for every pair $(X, Y)$ of horizontal vector fields, one has

$$
\beta_{f}(X, Y)=B_{f}(X, Y)+\left\langle\left(\frac{1}{2} J+\tau\right) X, Y\right\rangle_{G_{\theta}} d f(\xi)
$$

and

$$
H_{b}(f)=H(f)-B_{f}(\xi, \xi)=H(f)-\nabla_{\xi}^{f} d f(\xi)
$$


where $B_{f}(X, Y)=\nabla_{X}^{f} d f(Y)-d f\left(\nabla_{X}^{g_{\theta}} Y\right)$ and $H(f)=$ trace $_{g_{\theta}} B_{f}$ is the tension vector field (see [14]). In the particular case where $f$ is an isometric immersion from $\left(M, g_{\theta}\right)$ to $(N, h), B_{f}$ coincides with the second fundamental form of $f$ and $H(f)$ coincides with its mean curvature vector.

For the natural inclusion $j: \mathbb{S}^{2 n+1} \hookrightarrow \mathbb{C}^{n+1}$ of $\mathbb{S}^{2 n+1}$, the form $\beta_{j}$ is given by, $\beta_{j}(X, Y)=-\langle X, Y\rangle_{\mathbb{C}^{n+1}} \vec{x}+\langle J X, Y\rangle_{\mathbb{C}^{n+1}} J \vec{x}$, where $\vec{x}$ is the position vector field (here $v(x)=-\vec{x}$ and $\xi(x)=2 J \vec{x})$. Thus,

$$
H_{b}(j)=-2 n \vec{x}
$$

In the sequel we will focus on maps $f:(M, \theta) \longrightarrow(N, h)$ that preserve lengths in the horizontal directions as well as the orthogonality between $H(M)$ and $\xi$, that is, $\forall X \in H(M)$,

$$
|d f(X)|_{h}=|X|_{G_{\theta}} \quad \text { and } \quad\langle d f(X), d f(\xi)\rangle_{h}=0,
$$

which also amounts to $f^{*} h=g_{\theta}+(\mu-1) \theta^{2}$ for some nonnegative function $\mu$ on $M$. For convenience, such a map will be termed semi-isometric. Notice that the dimension of the target manifold $N$ should be at least $2 n$. When the dimension of $N$ is $2 n$, then a semi-isometric map $f:(M, \theta) \longrightarrow(N, h)$ is noting but a Riemannian submersion satisfying $d f(\xi)=0$. Important examples are given by the standard projection from the Heisenberg group $\mathbb{H} \mathbb{H}^{n}$ to $\mathbb{R}^{2 n}$ and the Hopf fibration $\mathbb{S}^{2 n+1} \rightarrow \mathbb{C} P^{n}$.

Lemma 2.1. Let $(M, \theta)$ be a strictly pseudoconvex $C R$ manifold and let $(N, h)$ be a Riemannian manifold. If $f:(M, \theta) \longrightarrow(N, h)$ is a $C^{2}$ semiisometric map, then the form $\beta_{f}$ takes its values in the orthogonal complement of $d f(H(M))$. In particular, the vector $H_{b}(f)$ is orthogonal to $d f(H(M))$.

Proof. Let $X, Y$ and $Z$ be three horizontal vector fields. Since the LeviCivita connection of $(N, h)$ is torsionless, one has $\nabla_{X}^{f} d f(Y)-\nabla_{Y}^{f} d f(X)=$ $d f([X, Y])$. From the properties of the torsion of the Tanaka-Webster connection $\nabla$, one has $\nabla_{X} Y-\nabla_{Y} X=[X, Y]^{H}$. Thus,

$$
\beta_{f}(X, Y)-\beta_{f}(Y, X)=\theta([X, Y]) d f(\xi) .
$$

Since $d f(\xi)$ is orthogonal to $d f(H(M))$, we deduce the following symmetry property:

$$
\left\langle\beta_{f}(X, Y), d f(Z)\right\rangle_{h}=\left\langle\beta_{f}(Y, X), d f(Z)\right\rangle_{h} .
$$

On the other hand, we have,

$$
Z \cdot\langle d f(X), d f(Y)\rangle_{h}=Z \cdot\langle X, Y\rangle_{G_{\theta}} .
$$

Since $G_{\theta}$ is parallel with respect to the Tanaka-Webster connection $\nabla$ and $h$ is parallel with respect to the Levi-Civita connection $\nabla^{h}$, one gets

$$
Z \cdot\langle d f(X), d f(Y)\rangle_{h}=\left\langle\nabla_{Z}^{f} d f(X), d f(Y)\right\rangle_{h}+\left\langle d f(X), \nabla_{Z}^{f} d f(Y)\right\rangle_{h}
$$

and

$$
\begin{aligned}
Z \cdot\langle X, Y\rangle_{G_{\theta}} & =\left\langle\nabla_{Z} X, Y\right\rangle_{G_{\theta}}+\left\langle X, \nabla_{Z} Y\right\rangle_{G_{\theta}} \\
& =\left\langle d f\left(\nabla_{Z} X\right), d f(Y)\right\rangle_{h}+\left\langle d f(X), d f\left(\nabla_{Z} Y\right)\right\rangle_{h}
\end{aligned}
$$


where the last equality comes from the fact that $\nabla_{Z} X$ and $\nabla_{Z} Y$ are horizontal. Replacing into (2.5) we obtain

$$
\left\langle\nabla_{Z}^{f} d f(X)-d f\left(\nabla_{Z} X\right), d f(Y)\right\rangle_{h}+\left\langle\nabla_{Z}^{f} d f(Y)-d f\left(\nabla_{Z} Y\right), d f(X)\right\rangle_{h}=0 .
$$

Therefore, $\forall X, Y, Z \in H(M)$,

$$
\left\langle\beta_{f}(Z, X), d f(Y)\right\rangle_{h}+\left\langle\beta_{f}(Z, Y), d f(X)\right\rangle_{h}=0 .
$$

Taking $X=Y$ in (2.6) we obtain, $\forall X, Z \in H(M)$,

$$
\left\langle\beta_{f}(Z, X), d f(X)\right\rangle_{h}=0 .
$$

Now, taking $Z=X$ in (2.6) and using (2.4) and (2.7), we get, $\forall X, Y \in$ $H(M)$,

$$
\left\langle\beta_{f}(X, X), d f(Y)\right\rangle_{h}=0 .
$$

The symmetry property (2.4) enables us to conclude.

A direct consequence of Lemma2.1 is the following

Corollary 2.1. If $f:(M, \theta) \longrightarrow(N, h)$ is a Riemannian submersion from a strictly pseudoconvex $C R$ manifold $(M, \theta)$ to a Riemannian manifold $(N, h)$ with $d f(\xi)=0$, then $\beta_{f}=0$ and $H_{b}(f)=0$.

\section{EigenVAlues of THE SUBlaPlacian AND SEMI-ISOMETRIC MAPS INTO}

\section{EUCLIDEAN SPACES}

Let $(M, \theta)$ be a strictly pseudoconvex CR manifold and let $\Omega$ be a bounded (relatively compact) domain of $M$. In the case where $M$ is a closed manifold, we allow $\Omega$ to be equal to the whole of $M$. We are interested in Schrödinger-type operator $-\Delta_{b}+V$ where $V$ is a function on $\Omega$. We assume in all the sequel that the spectrum of $-\Delta_{b}+V$ in $\Omega$, with Dirichlet boundary conditions if $\partial \Omega \neq \emptyset$, is discrete and bounded from below. We will always denote by $\left\{\lambda_{j}\right\}_{j \geq 1}$ the non decreasing sequence of eigenvalues of $-\Delta_{b}+V$ and by $\left\{u_{j}\right\}_{j \geq 1}$ a complete orthonormal family of eigenfunctions in $\Omega$ with $\left(-\Delta_{b}+V\right) u_{j}=\lambda_{j} u_{j}$.

Theorem 3.1. Let $(M, \theta)$ be a strictly pseudoconvex $C R$ manifold of real dimension $2 n+1$ and let $f:(M, \theta) \longrightarrow \mathbb{R}^{m}$ be a semi-isometric $C^{2}$ map. The sequence of eigenvalues $\left\{\lambda_{j}\right\}_{j \geq 1}$ of the Schrödinger-type operator $-\Delta_{b}+V$ in a bounded domain $\Omega \subset M$, with Dirichlet boundary conditions if $\Omega \neq M$, satisfies for every $k \geq 1$ and $p \in \mathbb{R}$,

$$
\sum_{i=1}^{k}\left(\lambda_{k+1}-\lambda_{i}\right)^{p} \leq \frac{\max \{2, p\}}{n} \sum_{i=1}^{k}\left(\lambda_{k+1}-\lambda_{i}\right)^{p-1}\left(\lambda_{i}+\frac{1}{4} D_{i}\right)
$$

with

$$
D_{i}=\int_{\Omega}\left(\left|H_{b}(f)\right|_{\mathbb{R}^{m}}^{2}-4 V\right) u_{i}^{2} \vartheta_{\theta}
$$


Moreover, if $V$ is bounded below on $\Omega$, then for every $k \geq 1$,

$$
\lambda_{k+1} \leq\left(1+\frac{2}{n}\right) \frac{1}{k} \sum_{i=1}^{k} \lambda_{i}+\frac{1}{2 n} D_{\infty}
$$

and

$$
\lambda_{k+1} \leq\left(1+\frac{2}{n}\right) k^{\frac{1}{n}} \lambda_{1}+\frac{1}{4}\left(\left(1+\frac{2}{n}\right) k^{\frac{1}{n}}-1\right) D_{\infty}
$$

with $D_{\infty}=\sup _{\Omega}\left(\left|H_{b}(f)\right|_{\mathbb{R}^{m}}^{2}-4 V\right)$.

Applying this result to the standard CR sphere whose standard embedding $j: \mathbb{S}^{2 n+1} \rightarrow \mathbb{C}^{n+1}$ satisfies $\left|H_{b}(j)\right|_{\mathbb{C}^{n+1}}^{2}=4 n^{2}$ (see (2.3) ), we get the following

Corollary 3.1. Let $\Omega$ be a domain in the standard CR sphere $\mathbb{S}^{2 n+1} \subset \mathbb{C}^{n+1}$. The eigenvalues of the operator $-\Delta_{b}+V$ in $\Omega$, with Dirichlet boundary conditions if $\Omega \neq \mathbb{S}^{2 n+1}$, satisfy, for every $k \geq 1$ and $p \in \mathbb{R}$,

$$
\sum_{i=1}^{k}\left(\lambda_{k+1}-\lambda_{i}\right)^{p} \leq \frac{\max \{2, p\}}{n} \sum_{i=1}^{k}\left(\lambda_{k+1}-\lambda_{i}\right)^{p-1}\left(\lambda_{i}+n^{2}-T_{i}\right)
$$

with $T_{i}=\int_{\Omega} V u_{i}^{2} \vartheta_{\theta}$. Moreover, if $V$ is bounded below on $\Omega$, then, for every $k \geq 1$,

$$
\lambda_{k+1} \leq\left(1+\frac{2}{n}\right) \frac{1}{k} \sum_{i=1}^{k} \lambda_{i}+2 n-\frac{2}{n} \inf _{\Omega} V
$$

and

$$
\lambda_{k+1} \leq\left(1+\frac{2}{n}\right) k^{\frac{1}{n}} \lambda_{1}+C(n, k, V)
$$

with $C(n, k, V)=\left(\left(1+\frac{2}{n}\right) k^{\frac{1}{n}}-1\right)\left(n^{2}-\inf _{\Omega} V\right)$.

Theorem 3.1 also applies to the Heisenberg group $\mathbb{H}^{n}$ endowed with its standard CR structure. The corresponding sub-Laplacian is nothing but the operator $\Delta_{\mathbb{H}^{n}}=\frac{1}{4} \sum_{j \leq n}\left(X_{j}^{2}+Y_{j}^{2}\right)$ (see section 5 for details). Since the standard projection $\mathbb{H}^{n} \rightarrow \mathbb{R}^{2 n}$ is semi-isometric (up to a dilation, see (5.3) below) with zero Levi-tension (see Corollary 2.1), Theorem 3.1 leads to the following corollary which improves the results by Niu-Zhang [26] and El Soufi-Harrell-Ilias [16].

Corollary 3.2. Let $\Omega$ be a domain in the Heisenberg group $\mathbb{H}^{n}$. The eigenvalues of the operator $-\Delta_{b}+V$ in $\Omega$, with Dirichlet boundary conditions, satisfy, for every $k \geq 1$ and $p \in \mathbb{R}$,

$$
\sum_{i=1}^{k}\left(\lambda_{k+1}-\lambda_{i}\right)^{p} \leq \frac{\max \{2, p\}}{n} \sum_{i=1}^{k}\left(\lambda_{k+1}-\lambda_{i}\right)^{p-1}\left(\lambda_{i}-T_{i}\right)
$$


with $T_{i}=\int_{\Omega} V u_{i}^{2} \vartheta_{\theta}$. Moreover, if $V$ is bounded below on $\Omega$, then, for every $k \geq 1$,

$$
\lambda_{k+1} \leq\left(1+\frac{2}{n}\right) \frac{1}{k} \sum_{i=1}^{k} \lambda_{i}-\frac{2}{n} \inf _{\Omega} V
$$

and

$$
\lambda_{k+1} \leq\left(1+\frac{2}{n}\right) k^{\frac{1}{n}} \lambda_{1}-\left(\left(1+\frac{2}{n}\right) k^{\frac{1}{n}}-1\right) \inf _{\Omega} V .
$$

The proof of Theorem 3.1 relies on a general result of algebraic nature using commutators. The use of this approach in obtaining bounds for eigenvalues is now fairly prevalent. Pioneering works in this direction are due to Harrell, alone or with collaborators (see [16, 18, 19, 20, 21, 22]). For our purpose, we will use the following version that can be found in a recent paper by Ashbaugh and Hermi [2] (see inequality (26) of Corollary 3 and inequality (46) of Corollary 8 in [2]).

Lemma 3.1. Let $A: \mathcal{D} \subset \mathcal{H} \rightarrow \mathcal{H}$ be a self-adjoint operator defined on a dense domain $\mathcal{D}$ which is semibounded below and has a discrete spectrum $\lambda_{1} \leq \lambda_{2} \cdots \leq \lambda_{i} \leq \cdots$. Let $B: A(\mathcal{D}) \rightarrow \mathcal{H}$ be a symmetric operator which leaves $\mathcal{D}$ invariant. Denoting by $\left\{u_{i}\right\}_{i \geq 1}$ a complete orthonormal family of eigenvectors of $A$ with $A u_{i}=\lambda_{i} u_{i}$, we have, for every $k \geq 1$ and $p \in \mathbb{R}$,

$$
\sum_{i=1}^{k}\left(\lambda_{k+1}-\lambda_{i}\right)^{p}\left\langle[A, B] u_{i}, B u_{i}\right\rangle \leq \max \left\{1, \frac{p}{2}\right\} \sum_{i=1}^{k}\left(\lambda_{k+1}-\lambda_{i}\right)^{p-1}\left\|[A, B] u_{i}\right\|^{2} .
$$

Proof of Theorem 3.1. Let $f:(M, \theta) \rightarrow \mathbb{R}^{m}$ be a semi-isometric map and let $f_{1}, \ldots, f_{m}$ be its Euclidean components. For each $\alpha=1, \ldots, m$, we denote by $f_{\alpha}$ the multiplication operator naturally associated with $f_{\alpha}$. Let us start by the calculation of $\left\langle\left[-\Delta_{b}+V, f_{\alpha}\right] u_{i}, f_{\alpha} u_{i}\right\rangle_{L^{2}}$ and $\left\|\left[-\Delta_{b}+V, f_{\alpha}\right] u_{i}\right\|_{L^{2}}^{2}$. One has,

$$
\begin{aligned}
{\left[-\Delta_{b}+V, f_{\alpha}\right] u_{i} } & =-\Delta_{b}\left(f_{\alpha} u_{i}\right)+f_{\alpha}\left(\Delta_{b} u_{i}\right) \\
& =-\left(\Delta_{b} f_{\alpha}\right) u_{i}-2\left\langle\nabla^{H} f_{\alpha}, \nabla^{H} u_{i}\right\rangle_{G_{\theta}} .
\end{aligned}
$$

Thus,

$$
\left\langle\left[-\Delta_{b}+V, f_{\alpha}\right] u_{i}, f_{\alpha} u_{i}\right\rangle_{L^{2}}=-\int_{\Omega} f_{\alpha}\left(\Delta_{b} f_{\alpha}\right) u_{i}^{2}-\frac{1}{2} \int_{\Omega}\left\langle\nabla^{H} f_{\alpha}^{2}, \nabla^{H} u_{i}^{2}\right\rangle_{G_{\theta}} .
$$

Here and in the sequel, all the integrals over $M$ are calculated with respect to the volume form $\vartheta_{\theta}$ or, equivalently, the Riemannian volume element induced by the Webster metric $g_{\theta}$. The integration over the eventual boundary is calculated with respect to the Riemannian metric induced on $\partial \Omega$ by the Webster metric $g_{\theta}$. Integration by parts leads to (see (2.1)

$$
\int_{\Omega}\left\langle\nabla^{H} f_{\alpha}^{2}, \nabla^{H} u_{i}^{2}\right\rangle_{G_{\theta}}=-\int_{\Omega}\left(\Delta_{b} f_{\alpha}^{2}\right) u_{i}^{2}+\int_{\partial M} u_{i}^{2}\left\langle\nabla^{H} f_{\alpha}^{2}, v\right\rangle_{g_{\theta}}
$$


where $v$ is the unit normal vector to the boundary with respect to the Webster metric $g_{\theta}$. Since $u_{i}$ vanishes on $\partial \Omega$ when $\partial \Omega \neq \emptyset$, we get

$$
\begin{aligned}
\int_{\Omega}\left\langle\nabla^{H} f_{\alpha}^{2}, \nabla^{H} u_{i}^{2}\right\rangle_{G_{\theta}} & =-\int_{\Omega}\left(\Delta_{b} f_{\alpha}^{2}\right) u_{i}^{2} \\
& =-2\left[\int_{\Omega} f_{\alpha}\left(\Delta_{b} f_{\alpha}\right) u_{i}^{2}+\int_{\Omega}\left|\nabla^{H} f_{\alpha}\right|_{G_{\theta}}^{2} u_{i}^{2}\right] .
\end{aligned}
$$

Substituting in (3.4) we obtain

$$
\left\langle\left[-\Delta_{b}+V, f_{\alpha}\right] u_{i}, f_{\alpha} u_{i}\right\rangle_{L^{2}}=\int_{\Omega}\left|\nabla^{H} f_{\alpha}\right|_{G_{\theta}}^{2} u_{i}^{2} .
$$

Thus

$$
\sum_{\alpha=1}^{m}\left\langle\left[-\Delta_{b}+V, f_{\alpha}\right] u_{i}, f_{\alpha} u_{i}\right\rangle_{L^{2}}=\sum_{\alpha=1}^{m} \int_{\Omega}\left|\nabla^{H} f_{\alpha}\right|_{G_{\theta}}^{2} u_{i}^{2} .
$$

Now, since $f$ preserves the Levi-form, one has with respect to a $G_{\theta}$-orthonormal frame $\left\{e_{i}\right\}$ of $H_{p}(M)$,

$$
\begin{aligned}
\sum_{\alpha=1}^{m}\left|\nabla^{H} f_{\alpha}\right|_{G_{\theta}}^{2} & =\sum_{\alpha=1}^{m} \sum_{i=1}^{2 n}\left\langle\nabla^{H} f_{\alpha}, e_{i}\right\rangle_{G_{\theta}}^{2}=\sum_{i=1}^{2 n} \sum_{\alpha=1}^{m}\left\langle\nabla f_{\alpha}, e_{i}\right\rangle_{G_{\theta}}^{2} \\
& =\sum_{i=1}^{2 n}\left|d f\left(e_{i}\right)\right|_{\mathbb{R}^{m}}^{2}=\sum_{i=1}^{2 n}\left|e_{i}\right|_{G_{\theta}}^{2}=2 n .
\end{aligned}
$$

Therefore,

$$
\sum_{\alpha=1}^{m}\left\langle\left[-\Delta_{b}+V, f_{\alpha}\right] u_{i}, f_{\alpha} u_{i}\right\rangle_{L^{2}}=2 n \int_{\Omega} u_{i}^{2}=2 n .
$$

On the other hand, we have

$$
\begin{aligned}
\left\|\left[-\Delta_{b}+V, f_{\alpha}\right] u_{i}\right\|_{L^{2}}^{2} & =\int_{\Omega}\left(\left(\Delta_{b} f_{\alpha}\right) u_{i}+2\left\langle\nabla^{H} f_{\alpha}, \nabla^{H} u_{i}\right\rangle_{G_{\theta}}\right)^{2} \\
& =\int_{\Omega}\left(\Delta_{b} f_{\alpha}\right)^{2} u_{i}^{2}+4 \int_{\Omega}\left\langle\nabla^{H} f_{\alpha}, \nabla^{H} u_{i}\right\rangle_{G_{\theta}}^{2} \\
& +2 \int_{\Omega}\left(\Delta_{b} f_{\alpha}\right)\left\langle\nabla^{H} f_{\alpha}, \nabla^{H} u_{i}^{2}\right\rangle_{G_{\theta}} .
\end{aligned}
$$

Using (2.2), we get

$$
\sum_{\alpha=1}^{m} \int_{\Omega}\left(\Delta_{b} f_{\alpha}\right)^{2} u_{i}^{2}=\int_{\Omega}\left|H_{b}(f)\right|_{\mathbb{R}^{m}}^{2} u_{i}^{2} .
$$

Using the isometry property of $f$ with respect to horizontal directions, we get

$$
\begin{aligned}
\sum_{\alpha=1}^{m}\left\langle\nabla^{H} f_{\alpha}, \nabla^{H} u_{i}\right\rangle_{G_{\theta}}^{2} & =\sum_{\alpha=1}^{m}\left\langle\nabla f_{\alpha}, \nabla^{H} u_{i}\right\rangle_{G_{\theta}}^{2}=\sum_{\alpha=1}^{m}\left|d f_{\alpha}\left(\nabla^{H} u_{i}\right)\right|_{\mathbb{R}^{m}}^{2} \\
& =\left|d f\left(\nabla^{H} u_{i}\right)\right|_{\mathbb{R}^{m}}^{2}=\left|\nabla^{H} u_{i}\right|_{G_{\theta}}^{2} .
\end{aligned}
$$


Thus,

$$
\sum_{\alpha=1}^{m} \int_{\Omega}\left\langle\nabla^{H} f_{\alpha}, \nabla^{H} u_{i}\right\rangle_{G_{\theta}}^{2}=\int_{\Omega}\left|\nabla^{H} u_{i}\right|_{G_{\theta}}^{2}=\lambda_{i}-\int_{\Omega} V u_{i}^{2} .
$$

Finally, denoting by $\left\{E_{\alpha}\right\}$ the standard basis of $\mathbb{R}^{m}$ and using Lemma 2.1 , we get,

$$
\begin{aligned}
\sum_{\alpha}^{m} \int_{\Omega} \Delta_{b} f_{\alpha}\left\langle\nabla^{H} f_{\alpha}, \nabla^{H} u_{i}^{2}\right\rangle_{G_{\theta}} & =\left\langle\sum_{\alpha}^{m} \Delta_{b} f_{\alpha} E_{\alpha}, \sum_{\alpha}^{m}\left\langle\nabla f_{\alpha}, \nabla^{H} u_{i}^{2}\right\rangle_{G_{\theta}} E_{\alpha}\right\rangle_{\mathbb{R}^{m}} \\
& =\left\langle H_{b}(f), d f\left(\nabla^{H} u_{i}^{2}\right)\right\rangle_{\mathbb{R}^{m}}=0 .
\end{aligned}
$$

Using all these facts, we get

$$
\sum_{\alpha=1}^{m}\left\|\left[-\Delta_{b}+V, f_{\alpha}\right] u_{i}\right\|_{L^{2}}^{2}=4\left(\lambda_{i}-\int_{\Omega} V u_{i}^{2}\right)+\int_{\Omega}\left|H_{b}(f)\right|_{\mathbb{R}^{m}}^{2} u_{i}^{2} .
$$

Applying Lemma 3.1 with $A=-\Delta_{b}+V$ and $B=f_{\alpha}$, summing up with respect to $\alpha=1, \ldots, m$, and using (3.5) and (3.6), we get the inequality (3.1).

To prove the inequality (3.2), we consider the quadratic relation that we derive from (3.1) after replacing $p$ by 2 and $D_{i}$ by $D_{\infty}$, that is, $\forall k \geq 1$,

$$
\sum_{i=1}^{k}\left(\lambda_{k+1}-\lambda_{i}\right)^{2} \leq \frac{2}{n} \sum_{i=1}^{k}\left(\lambda_{k+1}-\lambda_{i}\right)\left(\lambda_{i}+\frac{D_{\infty}}{4}\right)
$$

which leads to

$$
\lambda_{k+1}^{2}-\lambda_{k+1}\left(\left(2+\frac{2}{n}\right) M_{k}+\frac{1}{2 n} D_{\infty}\right)+\left(1+\frac{2}{n}\right) Q_{k}+\frac{1}{2 n} D_{\infty} M_{k} \leq 0
$$

with $M_{k}=\frac{1}{k} \sum_{i=1}^{k} \lambda_{i}$ and $Q_{k}=\frac{1}{k} \sum_{i=1}^{k} \lambda_{i}^{2}$. Using Cauchy-Schwarz inequality $M_{k}^{2} \leq Q_{k}$, we get

$$
\lambda_{k+1}^{2}-\lambda_{k+1}\left(\left(2+\frac{2}{n}\right) M_{k}+\frac{1}{2 n} D_{\infty}\right)+\left(1+\frac{2}{n}\right) M_{k}^{2}+\frac{1}{2 n} D_{\infty} M_{k} \leq 0
$$

which can also be written as follows:

$$
\left(\lambda_{k+1}-M_{k}\right)\left(\lambda_{k+1}-\left(1+\frac{2}{n}\right) M_{k}-\frac{1}{2 n} D_{\infty}\right) \leq 0 .
$$

Since $\lambda_{k+1}-M_{k}$ is clearly nonnegative, we get $\lambda_{k+1} \leq\left(1+\frac{2}{n}\right) M_{k}+\frac{1}{2 n} D_{\infty}$ which proves (3.2).

Now, if we set $\bar{\lambda}_{i}:=\lambda_{i}+\frac{1}{4} D_{\infty}$, then the inequality (3.7) reads

$$
\sum_{1}^{k}\left(\bar{\lambda}_{k+1}-\bar{\lambda}_{i}\right)^{2} \leq \frac{2}{n} \sum_{1}^{k}\left(\bar{\lambda}_{k+1}-\bar{\lambda}_{i}\right) \bar{\lambda}_{i}
$$

Following Cheng and Yang's argument [11, Theorem 2.1 and Corollary 2.1], we obtain

$$
\bar{\lambda}_{k+1} \leq\left(1+\frac{2}{n}\right) \bar{\lambda}_{1} k^{\frac{1}{n}}
$$


which gives immediately the last inequality of the theorem.

4. Applications to RiemanNian SUbmersions OVER SUbManifolds of the EUCLIDEAN SPACE

Let $(M, \theta)$ be a strictly pseudoconvex CR manifold and let $f:(M, \theta) \rightarrow N$ be a Riemannian submersion over a Riemannian manifold $N$ of dimension $2 n$. The manifold $N$ admits infinitely many isometric immersions into Euclidean spaces. For every integer $m \geq 2 n$ we denote by $\mathcal{I}\left(N, \mathbb{R}^{m}\right)$ the set of all $C^{2}$ isometric immersions from $N$ to the $m$-dimensional Euclidean space $\mathbb{R}^{m}$. Thanks to the Nash embedding theorem, the set $\cup_{m \in \mathbb{N}} \mathcal{I}\left(N, \mathbb{R}^{m}\right)$ is never empty, which motivates the introduction of the following invariant :

$$
H^{\text {euc }}(N)=\inf _{\phi \in \cup_{m \in \mathbb{N}} I\left(N, \mathbb{R}^{m}\right)}\|H(\phi)\|_{\infty}
$$

where $H(\phi)$ stands for the mean curvature vector field of $\phi$.

Theorem 4.1. Let $(M, \theta)$ be a strictly pseudoconvex $C R$ manifold of real dimension $2 n+1$ and let $f:(M, \theta) \rightarrow N$ be a Riemannian submersion over a Riemannian manifold of dimension $2 n$ such that $d f(\xi)=0$. The eigenvalues of the operator $-\Delta_{b}+V$ in a bounded domain $\Omega \subset M$, with Dirichlet boundary conditions if $\Omega \neq M$, satisfy for every $k \geq 1$ and $p \in \mathbb{R}$,

$$
\sum_{i=1}^{k}\left(\lambda_{k+1}-\lambda_{i}\right)^{p} \leq \frac{\max \{2, p\}}{n} \sum_{i=1}^{k}\left(\lambda_{k+1}-\lambda_{i}\right)^{p-1}\left(\lambda_{i}+\frac{1}{4} H^{\text {euc }}(N)^{2}-T_{i}\right)
$$

with $T_{i}=\int_{\Omega} V u_{i}^{2} \vartheta_{\theta}$. Moreover, if $V$ is bounded below on $\Omega$, then, for every $k \geq 1$,

$$
\lambda_{k+1} \leq\left(1+\frac{2}{n}\right) \frac{1}{k} \sum_{i=1}^{k} \lambda_{i}+\frac{1}{2 n} H^{e u c}(N)^{2}-\frac{2}{n} \inf _{\Omega} V
$$

and

$$
\lambda_{k+1} \leq\left(1+\frac{2}{n}\right) k^{\frac{1}{n}} \lambda_{1}+C
$$

with $C=\left(\left(1+\frac{2}{n}\right) k^{\frac{1}{n}}-1\right)\left(\frac{1}{4} H^{\text {euc }}(N)^{2}-\inf _{\Omega} V\right)$.

Proof. Let $\phi: N \rightarrow \mathbb{R}^{m}$ be any isometric immersion. It is straightforward to check that the map $\hat{f}=\phi \circ f:(M, \theta) \rightarrow \mathbb{R}^{m}$ is semi-isometric and that, $\forall X, Y \in H(M)$,

$$
\beta_{\hat{f}}(X, Y)=d \phi\left(\beta_{f}(X, Y)\right)+B_{\phi}(d f(X), d f(Y))=B_{\phi}(d f(X), d f(Y)),
$$

where $B_{\phi}$ stands for the second fundamental form of $\phi$ and where the last equality follows from Corollary 2.1. Now, from the assumptions on $f$, the differential of $f$ induces, for each $x \in M$, an isometry between $H_{x}(M)$ and $T_{f(x)} N$. Thus, if $X_{1}, \cdots, X_{2 n}$ is a local orthonormal frame of $H(M)$, then $d f\left(X_{1}\right), \cdots, d f\left(X_{2 n}\right)$ is also an orthonormal frame of $T N$. This leads to the equality

$$
H_{b}(\hat{f})=H(\phi)
$$


Therefore, it suffices to apply Theorem 3.1 to $\hat{f}$ and then take the infimum with respect to $\phi$ to finish the proof.

For example, when $N$ is an open set of $\mathbb{R}^{2 n}$ or, more generally, a minimal submanifold in $\mathbb{R}^{m}$, then $H^{\text {euc }}(N)=0$ and the Theorem above gives a class of pseudoconvex CR manifolds including domains of the Heisenberg group, for which the following holds :

Corollary 4.1. Let $(M, \theta)$ be a strictly pseudoconvex $C R$ manifold of real dimension $2 n+1$ which admits a Riemannian submersion $f:(M, \theta) \rightarrow N$ over a minimal submanifold $N$ of dimension $2 n$ of $\mathbb{R}^{m}$ such that $d f(\xi)=0$. The eigenvalues of the operator $-\Delta_{b}+V$ in a bounded domain $\Omega \subset M$, with Dirichlet boundary conditions if $\Omega \neq M$, satisfy for every $k \geq 1$ and $p \in \mathbb{R}$,

$$
\sum_{i=1}^{k}\left(\lambda_{k+1}-\lambda_{i}\right)^{p} \leq \frac{\max \{2, p\}}{n} \sum_{i=1}^{k}\left(\lambda_{k+1}-\lambda_{i}\right)^{p-1}\left(\lambda_{i}-T_{i}\right)
$$

with $T_{i}=\int_{\Omega} V u_{i}^{2} \vartheta_{\theta}$. Moreover, if $V$ is bounded below on $\Omega$, then for every $k \geq 1$,

$$
\lambda_{k+1} \leq\left(1+\frac{2}{n}\right) \frac{1}{k} \sum_{i=1}^{k} \lambda_{i}-\frac{2}{n} \inf _{\Omega} V
$$

and

$$
\lambda_{k+1} \leq\left(1+\frac{2}{n}\right) k^{\frac{1}{n}} \lambda_{1}-\left(\left(1+\frac{2}{n}\right) k^{\frac{1}{n}}-1\right) \inf _{\Omega} V .
$$

The natural embedding $j: \mathbb{S}^{2 n} \rightarrow \mathbb{R}^{2 n+1}$ of the sphere into the Euclidean space satisfies $|H(j)|_{\mathbb{R}^{2 n+1}}^{2}=4 n^{2}$. Thus, Theorem 4.1 leads to the following

Corollary 4.2. Let $(M, \theta)$ be a strictly pseudoconvex $C R$ manifold of real dimension $2 n+1$. Assume that $(M, \theta)$ admits a Riemannian submersion $f$ : $(M, \theta) \rightarrow D \subset \mathbb{S}^{2 n}$ over a domain $D$ of the standard sphere with $d f(\xi)=0$. The eigenvalues of the operator $-\Delta_{b}+V$ in a bounded domain $\Omega \subset M$, with Dirichlet boundary conditions if $\Omega \neq M$, satisfy for every $k \geq 1$ and $p \in \mathbb{R}$,

$$
\sum_{i=1}^{k}\left(\lambda_{k+1}-\lambda_{i}\right)^{p} \leq \frac{\max \{2, p\}}{n} \sum_{i=1}^{k}\left(\lambda_{k+1}-\lambda_{i}\right)^{p-1}\left(\lambda_{i}+n^{2}-T_{i}\right)
$$

with $T_{i}=\int_{\Omega} V u_{i}^{2} \vartheta_{\theta}$. Moreover, if $V$ is bounded below on $\Omega$, then for every $k \geq 1$,

$$
\lambda_{k+1} \leq\left(1+\frac{2}{n}\right) \frac{1}{k} \sum_{i=1}^{k} \lambda_{i}+2 n-\frac{2}{n} \inf _{\Omega} V
$$

and

$$
\lambda_{k+1} \leq\left(1+\frac{2}{n}\right) k^{\frac{1}{n}} \lambda_{1}+C
$$

with $C(n, k, V)=\left(\left(1+\frac{2}{n}\right) k^{\frac{1}{n}}-1\right)\left(n^{2}-\inf _{\Omega} V\right)$. 
In the particular case of a manifold $M$ without boundary that satisfies the assumptions of Corollary 4.2, one has, with $V=0, \lambda_{2}\left(-\Delta_{b}\right)=0$,

$$
\lambda_{2}\left(-\Delta_{b}\right) \leq 2 n
$$

and, for every $k \geq 1$,

$$
\lambda_{k+1}\left(-\Delta_{b}\right) \leq n(n+2) k^{\frac{1}{n}}-n^{2} .
$$

We denote by $\mathbb{F} P^{m}$ the $m$-dimensional real projective space if $\mathbb{F}=\mathbb{R}$, the complex projective space of real dimension $2 m$ if $\mathbb{F}=\mathbb{C}$, and the quaternionic projective space of real dimension $4 m$ if $\mathbb{F}=\mathbb{Q}$. The manifold $\mathbb{F} P^{m}$ carries a natural metric so that the Hopf fibration $\pi: \mathbb{S}^{d_{\mathbb{F}}(m+1)-1} \subset \mathbb{F}^{m+1} \rightarrow$ $\mathbb{F} P^{m}$ is a Riemannian fibration, where $d_{\mathbb{F}}=\operatorname{dim}_{\mathbb{R}} \mathbb{F}$.

Let $\mathcal{H}_{m+1}(\mathbb{F})=\left\{A \in \mathcal{M}_{m+1}(\mathbb{F}) \mid A^{*}:={ }^{t} A=A\right\}$ be the vector space of $(m+1) \times(m+1)$ Hermitian matrices with coefficients in $\mathbb{F}$, that we endow with the inner product

$$
\langle A, B\rangle=\frac{1}{2} \operatorname{trace}(A B) .
$$

The map $\psi: \mathbb{S}^{d_{\mathbb{F}}(m+1)-1} \subset \mathbb{F}^{m+1} \longrightarrow \mathcal{H}_{m+1}(\mathbb{F})$ given by

$$
\psi(z)=\left(\begin{array}{cccc}
\left|z_{0}\right|^{2} & z_{0} \bar{z}_{1} & \cdots & z_{0} \bar{z}_{m} \\
z_{1} \bar{z}_{0} & \left|z_{1}\right|^{2} & \cdots & z_{1} \bar{z}_{m} \\
\cdots & \cdots & \cdots & \cdots \\
z_{m} \bar{z}_{0} & z_{m} \bar{z}_{1} & \cdots & \left|z_{m}\right|^{2}
\end{array}\right)
$$

induces through the Hopf fibration an isometric embedding $\phi$ from $\mathbb{F} P^{m}$ into $\mathcal{H}_{m+1}(\mathbb{F})$. Moreover, $\phi\left(\mathbb{F} P^{m}\right)$ is a minimal submanifold of the hypersphere $\mathbb{S}\left(\frac{I}{m+1}, \sqrt{\frac{m}{2(m+1)}}\right)$ of $\mathcal{H}_{m+1}(\mathbb{F})$ of radius $\sqrt{\frac{m}{2(m+1)}}$ centered at $\frac{I}{m+1}$. One deduces that the mean curvature $H(\phi)$ satisfies

$$
|H(\phi)|^{2}=2 m(m+1) d_{\mathbb{F}}^{2} .
$$

Therefore, $H^{\text {euc }}\left(\mathbb{F} P^{m}\right)^{2} \leq 2 m(m+1) d_{\mathbb{F}}^{2}$ and Theorem 4.1 leads to the following

Corollary 4.3. Let $(M, \theta)$ be a strictly pseudoconvex $C R$ manifold of real dimension $2 n+1$ which admits a Riemannian submersion $f:(M, \theta) \rightarrow D \subset$ $\mathbb{F} P^{m}$ over a domain of the projective space $\mathbb{F} P^{m}$ of real dimension $2 n$ (i.e. $m=2 n / d_{\mathbb{F}}$ ) with $d f(\xi)=0$. The eigenvalues of the operator $-\Delta_{b}+V$ in a bounded domain $\Omega \subset M$, with Dirichlet boundary conditions if $\Omega \neq M$, satisfy for every $k \geq 1$ and $p \in \mathbb{R}$,

$$
\sum_{i=1}^{k}\left(\lambda_{k+1}-\lambda_{i}\right)^{p} \leq \frac{\max \{2, p\}}{n} \sum_{i=1}^{k}\left(\lambda_{k+1}-\lambda_{i}\right)^{p-1}\left(\lambda_{i}+n\left(2 n+d_{\mathbb{F}}\right)-T_{i}\right)
$$

with $T_{i}=\int_{\Omega} V u_{i}^{2} \vartheta_{\theta}$. Moreover, if $V$ is bounded below on $\Omega$, then for every $k \geq 1$,

$$
\lambda_{k+1} \leq\left(1+\frac{2}{n}\right) \frac{1}{k} \sum_{i=1}^{k} \lambda_{i}+2\left(2 n+d_{\mathbb{F}}\right)-\frac{2}{n} \inf _{\Omega} V
$$


and

$$
\lambda_{k+1} \leq\left(1+\frac{2}{n}\right) k^{\frac{1}{n}} \lambda_{1}+C
$$

with $C(n, k, V)=\left(\left(1+\frac{2}{n}\right) k^{\frac{1}{n}}-1\right)\left(n\left(2 n+d_{\mathbb{E}}\right)-\inf _{\Omega} V\right)$.

\section{EigenVAluES OF THE SUB-LAPLACIAN AND SEMI-ISOMETRIC MAPS INTO HEISENBERg GROUPS}

A model for the Heisenberg group is given by $\mathbb{H}^{m}=\mathbb{R}^{2 m+1} \cong \mathbb{C}^{m} \times \mathbb{R}$ endowed with the group law

$$
(z, t) \cdot(w, s)=(z+w, t+s+2 \operatorname{Im}\langle z, w\rangle),
$$

where $(z, t)=\left(z^{1}, \ldots, z^{n}, t\right),(w, s)=\left(w^{1}, \ldots, w^{n}, s\right) \in \mathbb{C}^{m} \times \mathbb{R}$, and $\langle z, w\rangle=$ $\sum_{j \leq m} z^{j} \bar{w}^{j}$ is the standard complex scalar product in $\mathbb{C}^{m}$. A natural basis of the corresponding Lie algebra is given by the family of left-invariant vector fields $\left\{X_{1}, \ldots, X_{m}, Y_{1}, \ldots, Y_{m}, T\right\}$ that coincides with the standard basis of $\mathbb{R}^{2 m+1}$ at the origin. That is, $T=\frac{\partial}{\partial t}$ and, $\forall j \leq m$,

$$
X_{j}=\frac{\partial}{\partial x_{j}}+2 y_{j} \frac{\partial}{\partial t}, \quad Y_{j}=\frac{\partial}{\partial y_{j}}-2 x_{j} \frac{\partial}{\partial t} .
$$

The Levi distribution $H\left(\mathbb{H}^{m}\right)$ is spanned by the vector fields $\left\{X_{j}, Y_{j}\right\}_{j \leq m}$. The complex sub-bundle $T^{1,0}$ of $T \mathbb{H}^{m} \otimes \mathbb{C}$ spanned by

$$
Z_{j}=\frac{\partial}{\partial z_{j}}+i \bar{z}_{j} \frac{\partial}{\partial t}=\frac{1}{2}\left(X_{j}-i Y_{j}\right), \quad j=1, \ldots m
$$

is such that $H\left(\mathbb{H}^{m}\right)=\operatorname{Re}\left(T^{1,0} \oplus T^{0,1}\right)$, with

$$
T^{0,1}=\operatorname{span}\left\{\bar{Z}_{j}=\frac{\partial}{\partial \bar{z}_{j}}-i z_{j} \frac{\partial}{\partial t}=\frac{1}{2}\left(X_{j}+i Y_{j}\right), j=1, \ldots m\right\} .
$$

This endows $H\left(\mathbb{H}^{m}\right)$ with an almost complex structure $J$ (so that $T^{1,0}=$ $\operatorname{ker}(J-i)$ and $\left.T^{0,1}=\operatorname{ker}(J+i)\right)$ which is actually integrable since $\left[Z_{j}, Z_{k}\right]=0$ for all $j, k \leq m$. Moreover, we have for all $j \leq m, J X_{j}=Y_{j}$.

The standard pseudo-Hermitian structure on $\mathbb{H}^{m}$ is

$$
\theta_{\mathbb{H}^{m}}=d t+i \sum_{j=1}^{m}\left(z^{j} d \bar{z}^{j}-\bar{z}^{j} d z^{j}\right)=d t+2 \sum_{j=1}^{m}\left(x^{j} d y^{j}-y^{j} d x^{j}\right),
$$

whose differential is $d \theta_{\mathbb{H}^{m}}=2 i \sum_{j=1}^{n} d z^{j} \wedge d \bar{z}^{j}$ and characteristic direction is $T=\frac{\partial}{\partial t}$. Since, for all $j \leq m$ and $k \leq m$, one has $\left[X_{j}, Y_{k}\right]=-4 \delta_{j k} T$ and $\left[X_{j}, X_{k}\right]=\left[Y_{j}, Y_{k}\right]=0$, the Levi form $G_{\theta_{\mathbb{H}^{m}}}$ on $H\left(\mathbb{H}^{m}\right)$ satisfies

$$
G_{\theta_{\mathbb{H}^{m}}}\left(X_{j}, X_{k}\right)=G_{\theta_{\mathbb{H}^{m}}}\left(Y_{j}, Y_{k}\right)=4 \delta_{j k} \quad \text { and } \quad G_{\theta_{\mathbb{H}^{m}}}\left(X_{j}, Y_{k}\right)=0 .
$$

We will denote by $g_{\mathbb{H}^{m}}$ the corresponding Webster metric. 
For a vector $W \in T_{(z, t)} \mathbb{H} \mathbb{H}^{m}$, if we denote by $\left\{v_{1}, w_{1}, \ldots, v_{n}, w_{n}, s\right\}$ its components with respect to the standard basis of $\mathbb{R}^{2 m+1}$, i.e.,

$$
W=\sum_{j=1}^{m} v_{j} \frac{\partial}{\partial x_{j}}+w_{j} \frac{\partial}{\partial y_{j}}+s \frac{\partial}{\partial t}
$$

then

$$
\begin{aligned}
W & =\sum_{j}\left(v_{j} X_{j}+w_{j} Y_{j}\right)+\left\{s+2 \sum_{j} w_{j} x_{j}-v_{j} y_{j}\right\} \frac{\partial}{\partial t} \\
& =\sum_{j}\left(v_{j} X_{j}+w_{j} Y_{j}\right)+\theta_{\mathbb{H}^{m}}(W) T .
\end{aligned}
$$

Hence, the coordinates of $W$ with respect to the basis $\left(X_{1}, Y_{1}, \ldots, X_{m}, Y_{m}, T\right)$ of $T_{(z, t)} \mathbb{H}^{m}$, are $\left\{v_{1}, w_{1}, \ldots, v_{n}, w_{n}, \theta_{\mathbb{H}^{m}}(W)\right\}$. Thus,

$$
\begin{aligned}
g_{\mathbb{H}^{m}}(W, W) & =4 \sum_{j}^{n}\left(v_{j}^{2}+w_{j}^{2}\right)+\theta_{\mathbb{H}^{m}}(W)^{2} \\
& =4|W|_{\mathbb{R}^{2 m+1}}^{2}-4 s^{2}+\theta_{\mathbb{H}^{m}}(W)^{2} .
\end{aligned}
$$

In particular, if $W$ is horizontal, then $g_{\mathbb{H}^{m}}(W, W)=4|W|_{\mathbb{R}^{2 m+1}}^{2}-4 s^{2}$.

Theorem 5.1. Let $(M, \theta)$ be a strictly pseudoconvex $C R$ manifold of dimension $2 n+1$ and let $f: M \longrightarrow \mathbb{H}^{m}$ be a $C^{2}$ semi-isometric map satisfying $d f(H(M)) \subseteq H\left(\mathbb{H}^{m}\right)$. Then the eigenvalues of the operator $-\Delta_{b}+V$ in any bounded domain $\Omega \subset M$, with Dirichlet boundary conditions if $\Omega \neq M$, satisfy for every $k \geq 1$ and $p \in \mathbb{R}$,

$$
\sum_{i=1}^{k}\left(\lambda_{k+1}-\lambda_{i}\right)^{p} \leq \frac{\max \{2, p\}}{n} \sum_{i=1}^{k}\left(\lambda_{k+1}-\lambda_{i}\right)^{p-1}\left(\lambda_{i}+\frac{1}{4} D_{i}\right)
$$

with

$$
D_{i}=\int_{\Omega}\left(\left|H_{b}(f)\right|_{\mathbb{H}^{m}}^{2}-4 V\right) u_{i}^{2} \vartheta_{\theta}
$$

Moreover, if $V$ is bounded below on $M$, then for every $k \geq 1$,

$$
\lambda_{k+1} \leq\left(1+\frac{2}{n}\right) \frac{1}{k} \sum_{i=1}^{k} \lambda_{i}+\frac{1}{2 n} D_{\infty}
$$

and

$$
\lambda_{k+1} \leq\left(1+\frac{2}{n}\right) k^{\frac{1}{n}} \lambda_{1}+\frac{1}{4}\left(\left(1+\frac{2}{n}\right) k^{\frac{1}{n}}-1\right) D_{\infty}
$$

with $D_{\infty}=\sup _{\Omega}\left(\left|H_{b}(f)\right|_{\mathbb{H}^{m}}^{2}-4 V\right)$.

In the particular case where $(M, \theta)$ is the Heisenberg group $\mathbb{H}^{n}$ endowed with the standard CR structure, this theorem provides an alternative way to derive Corollary 3.2

The following observation will be crucial for the proof of Theorem 5.1 . 
Proposition 5.1. Let $(M, \theta)$ be a strictly pseudoconvex $C R$ manifold and let

$$
\begin{aligned}
f: & (M, \theta) \longrightarrow \mathbb{H}^{m} \simeq \mathbb{C}^{m} \times \mathbb{R} \\
x & \longrightarrow f(x)=\left(F_{1}(x), \ldots, F_{m}(x), \alpha(x)\right)
\end{aligned}
$$

be a $C^{2}$ map such that $d f(H(M)) \subset H\left(\mathbb{H}^{m}\right)$. Then

$$
H_{b}(f)=\sum_{j=1}^{m}\left(\Delta_{b} \varphi_{j} X_{j}+\Delta_{b} \psi_{j} Y_{j}\right)
$$

where $\varphi_{j}(x)=\operatorname{ReF}_{j}(x)$ and $\psi_{j}(x)=\operatorname{Im} F_{j}(x)$.

In particular, $H_{b}(f)$ is a horizontal vector field and

$$
\left|H_{b}(f)\right|_{\mathbb{H}^{m}}^{2}=4 \sum_{j=1}^{m}\left[\left(\Delta_{b} \varphi_{j}\right)^{2}+\left(\Delta_{b} \psi_{j}\right)^{2}\right]
$$

Proof. One has, for any vector $W \in T M$,

$$
d f(W)=\sum_{j=1}^{m}\left(d \varphi_{j}(W) X_{j}+d \psi_{j}(W) Y_{j}\right)+\theta(d f(W)) T .
$$

For $W \in H(M), d f(W) \in H\left(\mathbb{H}^{m}\right)$ and, then,

$$
d f(W)=\sum_{j=1}^{m}\left(d \varphi_{j}(W) X_{j}+d \psi_{j}(W) Y_{j}\right) .
$$

Let $\left\{e_{i}\right\}$ be a local orthonormal frame of $H(M)$, then

$$
\beta_{f}\left(e_{i}, e_{i}\right)=\nabla_{e_{i}}^{f} d f\left(e_{i}\right)-d f\left(\nabla_{e_{i}} e_{i}\right) .
$$

Since $e_{i}$ and $\nabla_{e_{i}} e_{i}$ are horizontal and that $\left.d f(H(M)) \subset H(\mathbb{H}]^{m}\right)$, we have $\beta_{f}\left(e_{i}, e_{i}\right)=\sum_{j=1}^{m} \nabla_{e_{i}}^{f}\left(d \varphi_{j}\left(e_{i}\right) X_{j}+d \psi_{j}\left(e_{i}\right) Y_{j}\right)-\sum_{j=1}^{m}\left[d \varphi_{j}\left(\nabla_{e_{i}} e_{i}\right) X_{j}+d \psi_{j}\left(\nabla_{e_{i}} e_{i}\right) Y_{j}\right]$

with

and

$$
\nabla_{e_{i}}^{f}\left(d \varphi_{j}\left(e_{i}\right) X_{j}\right)=e_{i} \cdot d \varphi_{j}\left(e_{i}\right) X_{j}+d \varphi_{j}\left(e_{i}\right) \nabla_{d f\left(e_{i}\right)}^{\mathbb{H}^{m}} X_{j}
$$

Therefore,

$$
\nabla_{e_{i}}^{f}\left(d \psi_{j}\left(e_{i}\right) Y_{j}\right)=e_{i} \cdot d \psi_{j}\left(e_{i}\right) Y_{j}+d \psi_{j}\left(e_{i}\right) \nabla_{d f\left(e_{i}\right)}^{\mathbb{H}^{m}} Y_{j}
$$

$$
\begin{aligned}
\beta_{f}\left(e_{i}, e_{i}\right) & =\sum_{j=1}^{m}\left[e_{i} \cdot d \varphi_{j}\left(e_{i}\right)-d \varphi_{j}\left(\nabla_{e_{i}} e_{i}\right)\right] X_{j}+\sum_{j=1}^{m}\left[e_{i} \cdot d \psi_{j}\left(e_{i}\right)-d \psi_{j}\left(\nabla_{e_{i}} e_{i}\right)\right] Y_{j} \\
& +\sum_{j=1}^{m}\left[d \varphi_{j}\left(e_{i}\right) \nabla_{d f\left(e_{i}\right)}^{\mathbb{H}^{m}} X_{j}+d \psi_{j}\left(e_{i}\right) \nabla_{d f\left(e_{i}\right)}^{\mathbb{H}^{m}} Y_{j}\right]
\end{aligned}
$$

Recall that the Levi-Civita connection of $\mathbb{H}^{m}$ is such that

$$
\begin{gathered}
\nabla_{X_{k}}^{\mathbb{H}^{m}} X_{j}=\nabla_{Y_{k}}^{\mathbb{H}^{m}} Y_{j}=\nabla_{T}^{\mathbb{H}^{m}} T=0, \\
\nabla_{X_{k}}^{\mathbb{H}^{m}} Y_{j}=-2 \delta_{k j} T, \quad \nabla_{X_{k}}^{\mathbb{H}^{m}} T=2 Y_{k}, \quad \nabla_{Y_{k}}^{\mathbb{H}^{m}} T=-2 X_{k}, \\
\nabla_{Y_{k}}^{\mathbb{H}^{m}} X_{j}=2 \delta_{k j} T, \quad \nabla_{T}^{\mathbb{H}^{m}} X_{k}=2 Y_{k}, \quad \nabla_{T}^{\mathbb{H}^{m}} Y_{k}=-2 X_{k} .
\end{gathered}
$$


Thus,

$$
\begin{aligned}
\nabla_{d f\left(e_{i}\right)}^{\mathbb{H}^{m}} X_{j} & =\sum_{k}\left(d \varphi_{k}\left(e_{i}\right) \nabla_{X_{k}} X_{j}+d \psi_{k}\left(e_{i}\right) \nabla_{Y_{k}} X_{j}\right) \\
& =d \psi_{j}\left(e_{i}\right) \nabla_{Y_{j}} X_{j}=2 d \psi_{j}\left(e_{i}\right) T
\end{aligned}
$$

and

$$
\nabla_{d f\left(e_{i}\right)}^{\mathbb{H}^{m}} Y_{j}=-2 d \varphi_{j}\left(e_{i}\right) T
$$

Replacing into (5.9) and summing up with respect to $i$, we get

$$
\begin{aligned}
H_{b}(f) & =\sum_{i=1}^{2 n} \sum_{j=1}^{m}\left(\left[e_{i} \cdot d \varphi_{j}\left(e_{i}\right)-d \varphi_{j}\left(\nabla_{e_{i}} e_{i}\right)\right] X_{j}+\left[e_{i} \cdot d \psi_{j}\left(e_{i}\right)-d \psi_{j}\left(\nabla_{e_{i}} e_{i}\right)\right] Y_{j}\right) \\
& =\sum_{j=1}^{m}\left(\Delta_{b} \varphi_{j} X_{j}+\Delta_{b} \psi_{j} Y_{j}\right) .
\end{aligned}
$$

Proof of Theorem 5.1] As in the proof of Theorem 3.1, we will use the components of the map $f$ as multiplication operators. Let us write $f(x)=$ $\left(F_{1}(x), \ldots, F_{m}(x), \alpha(x)\right) \in \mathbb{C}^{m} \times \mathbb{R}$ and $F_{j}(x)=\varphi_{j}(x)+i \psi_{j}(x)$. The main difference with respect to the Euclidean case is that here, only the $\mathbb{C}^{m}$ components of $f$ come in. All along this proof we will use the fact that, $\forall W \in H_{x}(M)$, the vector $d f(W)$ is horizontal and (see (5.8))

$$
|d f(W)|_{\mathbb{H}^{m}}^{2}=4 \sum_{j=1}^{m}\left(\left|d \varphi_{j}(W)\right|^{2}+\left|d \psi_{j}(W)\right|^{2}\right) .
$$

Repeating the same calculations as in the proof of the Theorem 3.1, we get

$$
\begin{aligned}
\sum_{j=1}^{m}\left\langle\left[-\Delta_{b}+V, \varphi_{j}\right] u_{i}, \varphi_{j} u_{i}\right\rangle_{L^{2}} & +\left\langle\left[-\Delta_{b}+V, \psi_{j}\right] u_{i}, \psi_{j} u_{i}\right\rangle_{L^{2}} \\
& =\sum_{j=1}^{m} \int_{\Omega}\left\{\left|\nabla^{H} \varphi_{j}\right|_{G_{\theta}}^{2}+\left|\nabla^{H} \psi_{j}\right|_{G_{\theta}}^{2}\right\} u_{i}^{2} .
\end{aligned}
$$

Let $\left\{e_{i}\right\}$ be a $G_{\theta}$-orthonormal basis of $H_{x}(M)$, then

$$
\begin{aligned}
\sum_{j=1}^{m}\left|\nabla^{H} \varphi_{j}\right|_{G_{\theta}}^{2}+\left|\nabla^{H} \psi_{j}\right|_{G_{\theta}}^{2} & =\sum_{j=1}^{m} \sum_{i=1}^{2 n}\left\langle\nabla^{H} \varphi_{j}, e_{i}\right\rangle_{G_{\theta}}^{2}+\left\langle\nabla^{H} \psi_{j}, e_{i}\right\rangle_{G_{\theta}}^{2} \\
& =\sum_{i=1}^{2 n} \sum_{j=1}^{m}\left\langle\nabla \varphi_{j}, e_{i}\right\rangle_{G_{\theta}}^{2}+\left\langle\nabla \psi_{j}, e_{i}\right\rangle_{G_{\theta}}^{2} \\
& =\sum_{i=1}^{2 n} \sum_{j=1}^{2 m}\left(d \varphi_{j}\left(e_{i}\right)^{2}+d \psi_{j}\left(e_{i}\right)^{2}\right) \\
& =\frac{1}{4} \sum_{i=1}^{2 n}\left|d f\left(e_{i}\right)\right|_{\mathbb{H}^{m}}^{2}=\frac{n}{2} .
\end{aligned}
$$


Thus,

$$
\sum_{j=1}^{m}\left\langle\left[-\Delta_{b}+V, \varphi_{j}\right] u_{i}, \varphi_{j} u_{i}\right\rangle_{L^{2}}+\left\langle\left[-\Delta_{b}+V, \psi_{j}\right] u_{i}, \psi_{j} u_{i}\right\rangle_{L^{2}}=\frac{n}{2}
$$

On the other hand,

$$
\begin{aligned}
\left\|\left[-\Delta_{b}+V, \varphi_{j}\right] u_{i}\right\|_{L^{2}}^{2} & =\int_{\Omega}\left(\left(\Delta_{b} \varphi_{j}\right) u_{i}+2\left\langle\nabla^{H} \varphi_{j}, \nabla^{H} u_{i}\right\rangle_{G_{\theta}}\right)^{2} \\
& =\int_{\Omega}\left(\Delta_{b} \varphi_{j}\right)^{2} u_{i}^{2}+4 \int_{\Omega}\left\langle\nabla^{H} \varphi_{j}, \nabla^{H} u_{i}\right\rangle_{G_{\theta}}^{2} \\
& +2 \int_{\Omega}\left(\Delta_{b} \varphi_{j}\right)\left\langle\nabla^{H} \varphi_{j}, \nabla^{H} u_{i}^{2}\right\rangle_{G_{\theta}} .
\end{aligned}
$$

We have a similar formula for $\left\|\left[-\Delta_{b}+V, \psi_{j}\right] u_{i}\right\|_{L^{2}}^{2}$. Since $\nabla^{H} u_{i} \in H(M)$, one has

$$
\begin{aligned}
\sum_{j=1}^{m}\left\langle\nabla^{H} \varphi_{j}, \nabla^{H} u_{i}\right\rangle_{G_{\theta}}^{2} & +\left\langle\nabla^{H} \psi_{j}, \nabla^{H} u_{i}\right\rangle_{G_{\theta}}^{2} \\
& =\sum_{j=1}^{m}\left\{d \varphi_{j}\left(\nabla^{H} u_{i}\right)^{2}+d \psi_{j}\left(\nabla^{H} u_{i}\right)^{2}\right\} \\
& =\frac{1}{4}\left|d f\left(\nabla^{H} u_{i}\right)^{2}\right|_{\mathbb{H}^{m}}=\frac{1}{4}\left|\nabla^{H} u_{i}\right|_{G_{\theta}}^{2} .
\end{aligned}
$$

Therefore,

$$
\begin{aligned}
\sum_{j=1}^{m} \int_{\Omega}\left(\left\langle\nabla^{H} \varphi_{j}, \nabla^{H} u_{i}\right\rangle_{G_{\theta}}^{2}+\left\langle\nabla^{H} \psi_{j}, \nabla^{H} u_{i}\right\rangle_{G_{\theta}}^{2}\right) & =\frac{1}{4} \int_{\Omega}\left|\nabla^{H} u_{i}\right|_{G_{\theta}}^{2} \\
& =\frac{1}{4}\left(\lambda_{i}-\int_{\Omega} V u_{i}^{2}\right) .
\end{aligned}
$$

For the two remaining terms, we have thanks to Proposition 5.1 and the identity (5.3),

$$
\sum_{j=1}^{m} \int_{\Omega}\left(\left(\Delta_{b} \varphi_{j}\right)^{2}+\left(\Delta_{b} \psi_{j}\right)^{2}\right) u_{i}^{2}=\frac{1}{4} \int_{\Omega}\left|H_{b}(f)\right|_{\mathbb{H}^{m}}^{2} u_{i}^{2}
$$

and

$$
\begin{aligned}
\sum_{j=1}^{m} \int_{\Omega} & \left(\Delta_{b} \varphi_{j}\left\langle\nabla^{H} \varphi_{j}, \nabla^{H} u_{i}^{2}\right\rangle_{G_{\theta}}+\Delta_{b} \psi_{j}\left\langle\nabla^{H} \psi_{j}, \nabla^{H} u_{i}^{2}\right\rangle_{G_{\theta}}\right) \\
= & \frac{1}{4} \int_{\Omega}\left\langle H_{b}(f), \sum_{j=1}^{m} d \varphi_{j}\left(\nabla^{H} u_{i}^{2}\right) X_{j}+\sum_{j=1}^{m} d \psi_{j}\left(\nabla^{H} u_{i}^{2}\right) Y_{j}\right\rangle_{\mathbb{H}^{m}} \\
= & \frac{1}{4} \int_{\Omega}\left\langle H_{b}(f), d f\left(\nabla^{H} u_{i}^{2}\right)\right\rangle_{\mathbb{H}^{m}}=0
\end{aligned}
$$


where the last equality follows from the fact that $H_{b}(f)$ is orthogonal to $d f(H(M))$ (Lemma2.1). Finally,

$$
\left\|\left[-\Delta_{b}+V, \varphi_{j}\right] u_{i}\right\|_{L^{2}}^{2}+\left\|\left[-\Delta_{b}+V, \psi_{j}\right] u_{i}\right\|_{L^{2}}^{2}=\lambda_{i}+\frac{1}{4} \int_{\Omega}\left(\left|H_{b}(f)\right|_{\mathbb{H}^{m}}^{2}-V\right) u_{i}^{2} .
$$

Applying Lemma 3.1 with $A=-\Delta_{b}+V$ and $B=\varphi_{j}$ then $B=\psi_{j}$, summing up with respect to $j$ and using (5.11) and (5.12), we obtain the inequality (5.5).

As in the proof of Theorem 3.1, we derive the inequalities (5.6) and (5.7) from (5.5) with $p=2$.

6. ReILly tYPE INEQUALITIES FOR CR MANIFOLDS MAPPED INTO THE EUCLIDEAN SPACE OR THE HEISENBERG GROUP

Let $(M, \theta)$ be a compact strictly pseudo-convex $\mathrm{CR}$ manifold. If $f$ : $(M, \theta) \longrightarrow \mathbb{R}^{m}$ is a semi-isometric $C^{2}$ map, then Theorem 3.1 (i.e. inequality (3.1) with $k=1$ and $p=1$ ) gives,

$$
\lambda_{2}\left(-\Delta_{b}+V\right) \leq\left(1+\frac{2}{n}\right) \lambda_{1}\left(-\Delta_{b}+V\right)+\frac{1}{2 n} \int_{M}\left(\left|H_{b}(f)\right|_{\mathbb{R}^{m}}^{2}-4 V\right) u_{1}^{2} .
$$

When $M$ is a compact manifold without boundary and $V=0$, one has $\lambda_{1}\left(-\Delta_{b}\right)=0$ and $u_{1}^{2}=\frac{1}{V(M, \theta)}$. Therefore, the following Reilly type result holds (see[15] for details about Reilly inequalities)

$$
\lambda_{2}\left(-\Delta_{b}\right) \leq \frac{1}{2 n V(M, \theta)} \int_{M}\left|H_{b}(f)\right|_{\mathbb{R}^{m}}^{2} .
$$

This result can be obtained in an independent and simpler way, in the spirit of Reilly's proof, under weaker assumptions on $f$. Moreover, the equality case can be characterized. Indeed, we first have the following

Theorem 6.1. Let $(M, \theta)$ be a compact strictly pseudoconvex $C R$ manifold of dimension $2 n+1$ without boundary. For every $C^{2} \operatorname{map} f:(M, \theta) \longrightarrow \mathbb{R}^{m}$ one has

$$
\lambda_{2}\left(-\Delta_{b}\right) E_{b}(f) \leq \frac{1}{2} \int_{M}\left|H_{b}(f)\right|_{\mathbb{R}^{m}}^{2}
$$

where the equality holds if and only if the Euclidean components $f_{1}, \ldots, f_{m}$ of $f$ satisfy $-\Delta_{b} f_{\alpha}=\lambda_{2}\left(-\Delta_{b}\right)\left(f_{\alpha}-f f_{\alpha}\right)$ for every $\alpha \leq m$.

Proof. Replacing if necessary $f_{\alpha}$ by $f_{\alpha}-f f_{\alpha}$ we can assume without loss of generality that the Euclidean components $f_{1}, \ldots, f_{m}$ of $f$ satisfy $\int_{M} f_{\alpha} \vartheta_{\theta}=0$ so that, we have

$$
\lambda_{2}\left(-\Delta_{b}\right) \int_{M} f_{\alpha}^{2} \leq \int_{M}\left|\nabla^{H} f_{\alpha}\right|_{G_{\theta}}^{2} .
$$

Summing up with respect to $\alpha$, we get

$$
\lambda_{2}\left(-\Delta_{b}\right) \int_{M}|f|_{\mathbb{R}^{m}}^{2} \leq \int_{M} \sum_{\alpha=1}^{m}\left|\nabla^{H} f_{\alpha}\right|_{G_{\theta}}^{2} .
$$


Denoting by $\left\{\epsilon_{\alpha}\right\}$ the standard basis of $\mathbb{R}^{m}$ and by $\left\{X_{i}\right\}$ a local orthonormal frame of $H(M)$, we observe that

$$
\begin{aligned}
2 e_{b}(f) & =\sum_{i=1}^{2 n}\left|d f\left(X_{i}\right)\right|_{\mathbb{R}^{m}}^{2}=\sum_{i=1}^{2 n} \sum_{\alpha=1}^{m}\left\langle d f\left(X_{i}\right), \epsilon_{\alpha}\right\rangle_{\mathbb{R}^{m}}^{2} \\
& =\sum_{\alpha=1}^{m} \sum_{i=1}^{2 n}\left|d f_{\alpha}\left(X_{i}\right)\right|_{\mathbb{R}^{m}}^{2}=\sum_{\alpha=1}^{m}\left|\nabla^{H} f_{\alpha}\right|_{G_{\theta}}^{2} .
\end{aligned}
$$

Therefore,

$$
\lambda_{2}\left(-\Delta_{b}\right) \int_{M}|f|_{\mathbb{R}^{m}}^{2} \leq \int_{M} \sum_{\alpha=1}^{m}\left|\nabla^{H} f_{\alpha}\right|_{G_{\theta}}^{2}=2 E_{b}(f) .
$$

On the other hand, we have

$$
\begin{aligned}
4 E_{b}(f)^{2} & =\left(\sum_{\alpha=1}^{m} \int_{M}\left|\nabla^{H} f_{\alpha}\right|_{G_{\theta}}^{2}\right)^{2}=\left(\sum_{\alpha=1}^{m} \int_{M} f_{\alpha} \Delta_{b} f_{\alpha}\right)^{2} \\
& =\left(\int_{M}\left\langle f(x), \sum_{\alpha}^{m}\left(\Delta_{b} f_{\alpha}\right) \epsilon_{\alpha}\right\rangle_{\mathbb{R}^{m}}\right)^{2} \\
& =\left(\int_{M}\left\langle f(x), H_{b}(f)\right\rangle_{\mathbb{R}^{m}}\right)^{2} \leq \int_{M}|f|_{\mathbb{R}^{m}}^{2} \int_{M}\left|H_{b}(f)\right|_{\mathbb{R}^{m}}^{2} .
\end{aligned}
$$

Combining with (6.3), we get

$$
4 E_{b}(f)^{2} \leq \frac{2 E_{b}(f)}{\lambda_{2}\left(-\Delta_{b}\right)} \int_{M}\left|H_{b}(f)\right|_{\mathbb{R}^{m}}^{2}
$$

which gives the desired inequality.

Now, if we have, for every $\alpha \leq m,-\Delta_{b} f_{\alpha}=\lambda_{2}\left(-\Delta_{b}\right) f_{\alpha}$, then $H_{b}(f)=$ $\left(\Delta_{b} f_{1}, \ldots, \Delta_{b} f_{m}\right)=-\lambda_{2}\left(-\Delta_{b}\right) f$ and $\int_{M}\left|H_{b}(f)\right|_{\mathbb{R}^{m}}^{2}=\lambda_{2}\left(-\Delta_{b}\right)^{2} \int_{M}|f|_{\mathbb{R}^{m}}^{2}$. On the other hand, $E_{b}(f)=\int_{M} \sum_{\alpha=1}^{m}\left|\nabla^{H} f_{\alpha}\right|_{G_{\theta}}^{2}=\lambda_{2}\left(-\Delta_{b}\right) \int_{M}|f|_{\mathbb{R}^{m}}^{2}$ which implies that the equality holds in (6.1). Reciprocally, if the equality holds in (6.1) for a nonconstant map $f$, then it also holds in (6.2) for each $\alpha$. Thus, the functions $f_{1}, \ldots, f_{m}$ belong to the $\lambda_{2}\left(-\Delta_{b}\right)$-eigenspace of $-\Delta_{b}$.

If a map $f:(M, \theta) \longrightarrow \mathbb{R}^{m}$ preserves the metric with respect to horizontal directions (i.e., $|d f(X)|_{\mathbb{R}^{m}}=|X|_{G_{\theta}}$ for any $X \in H(M)$ ), then its energy density $e_{b}(f)$ is constant equal to $n$ and

$$
E_{b}(f)=n V(M, \theta) .
$$

Inequality 6.1) becomes in this case

$$
\lambda_{2}\left(-\Delta_{b}\right) \leq \frac{1}{2 n V(M, \theta)} \int_{M}\left|H_{b}(f)\right|_{\mathbb{R}^{m}}^{2} .
$$

The characterization of the equality case is the last inequality requires the following Takahashi's type result. 
Lemma 6.1. Let $(M, \theta)$ be a strictly pseudoconvex $C R$ manifold of dimension $2 n+1$ and let $f:(M, \theta) \longrightarrow \mathbb{R}^{m}$ be $C^{2}$ map.

i) Assume that $f(M)$ is contained in a sphere $\mathbb{S}^{m-1}(r)$ of radius $r$ centered at the origin. Then $f$ is pseudo-harmonic from $(M, \theta)$ to $S^{m-1}(r)$ if and only if its Euclidean components $f_{1}, \ldots, f_{m}$ satisfy, $\forall \alpha \leq m$,

$$
-\Delta_{b} f_{\alpha}=\mu f_{\alpha}
$$

with $\mu=\frac{2}{r^{2}} e_{b}(f) \in C^{\infty}(M)$.

ii) Assume that $f$ is semi-isometric. If the Euclidean components $f_{1}, \ldots, f_{m}$ of $f$ satisfy, $\forall \alpha \leq m,-\Delta_{b} f_{\alpha}=\lambda f_{\alpha}$, for some $\lambda \in \mathbb{R}$, then $f(M)$ is contained in the sphere $\mathbb{S}^{m-1}(r)$ of radius $r=\sqrt{\frac{2 n}{\lambda}}$ and $f$ is a pseudo-harmonic map from $(M, \theta)$ to $S^{m-1}(r)$. Conversely, if $f(M)$ is contained in a sphere $\mathbb{S}^{m-1}(r)$ and if $f$ is a pseudo-harmonic map from $(M, \theta)$ to $S^{m-1}(r)$, then, $\forall \alpha \leq m,-\Delta_{b} f_{\alpha}=\frac{2 n}{r^{2}} f_{\alpha}$.

This lemma is to be compared with Example 5.3 of [6] in which a sign mistake in Greenleaf's formula led to an incorrect characterization of pseudoharmonic maps into spheres.

Proof of Lemma 6.1 i) For convenience, let us write $f=j \circ \bar{f}$ where $j$ : $\mathbb{S}^{m-1}(r) \rightarrow \mathbb{R}^{m}$ is the standard embedding and $\bar{f}: M \rightarrow \mathbb{S}^{m-1}(r)$ is defined by $\bar{f}(x)=f(x)$. It is straightforward to observe that, $\forall X, Y \in H(M)$,

$$
\beta_{f}(X, Y)=B_{j}(d \bar{f}(X), d \bar{f}(Y))+d j\left(\beta_{\bar{f}}(X, Y)\right)
$$

where $B_{j}(W, W)=-\frac{1}{r^{2}}|W|_{\mathbb{R}^{m}}^{2} \vec{x}$ is the second fundamental form of the sphere $\mathbb{S}^{m-1}(r)$. Taking the trace, we obtain

$$
H_{b}(f)=-\frac{2 e_{b}(\bar{f})}{r^{2}} \bar{f}+d j\left(H_{b}(\bar{f})\right)=-\frac{2 e_{b}(f)}{r^{2}} f+d j\left(H_{b}(\bar{f})\right) .
$$

Hence, if $f$ is pseudo-harmonic from $(M, \theta)$ to $S^{m-1}(r)$, then $H_{b}(\bar{f})=0$ and, consequently, $H_{b}(f)=-\frac{2 e_{b}(f)}{r^{2}} f$ with $H_{b}(f)=\left(\Delta_{b} f_{1}, \ldots, \Delta_{b} f_{m}\right)$ (see (2.2)). Thus, $\forall \alpha \leq m,-\Delta_{b} f_{\alpha}=\frac{2}{r^{2}} e_{b}(f) f_{\alpha}$.

Reciprocally, if there exists a function $\mu \in C^{\infty}(M)$ such that $-\Delta_{b} f_{\alpha}=\mu f_{\alpha}$ for every $\alpha \leq m$, then

$$
0=\Delta_{b}\left(\sum_{\alpha=1}^{m} f_{\alpha}^{2}\right)=-2 \mu \sum_{\alpha=1}^{m} f_{\alpha}^{2}+2 \sum_{\alpha=1}^{m}\left|\nabla^{H} f_{\alpha}\right|_{G_{\theta}}^{2}=-2 \mu r^{2}+4 e_{b}(f) .
$$

Hence, $\mu=\frac{2 e_{b}(f)}{r^{2}}, H_{b}(f)=-\frac{2 e_{b}(f)}{r^{2}} f$ and, then, $H_{b}(\bar{f})=0$, which means that $f$ is pseudo-harmonic from $(M, \theta)$ to $S^{m-1}(r)$.

ii) From the assumptions, one has $H_{b}(f)=-\lambda f$ (see (2.2)). Since $f$ is semi-isometric, we know that $H_{b}(f)$ is orthogonal to $d f(H(M)$ ) (Lemma 2.1). Therefore, $\forall x \in M$ and $\forall X \in H_{x}(M)$, one has $\left\langle f(x), d f_{x}(X)\right\rangle_{\mathbb{R}^{m}}=0$ which implies that the function $x \mapsto|f(x)|_{\mathbb{R}^{m}}^{2}$ has zero derivative with respect to all horizontal directions. Since the distribution $H(M)$ is not integrable, 
this implies that $|f(x)|_{\mathbb{R}^{m}}^{2}$ is constant on $M$, that is $f(M)$ is contained in a sphere $\mathbb{S}^{m-1}(r)$ of radius $r$ centered at the origin. The pseudo-harmonicity of $f$ from $M$ into $\mathbb{S}^{m-1}(r)$ then follows from (i). Moreover, one necessarily has $\lambda=\frac{2 e_{b}(f)}{r^{2}}$ with $e_{b}(f)=n$ since $f$ is semi-isometric. Thus, the radius of the sphere is such that $r^{2}=\frac{2 n}{\lambda}$.

Theorem 6.1 and Lemma 6.1 lead to the following

Corollary 6.1. Let $(M, \theta)$ be a compact strictly pseudoconvex $C R$ manifold of dimension $2 n+1$ without boundary and let $f:(M, \theta) \longrightarrow \mathbb{R}^{m}$ be $C^{2}$ semi-isometric map. Then

$$
\lambda_{2}\left(-\Delta_{b}\right) \leq \frac{1}{2 n V(M, \theta)} \int_{M}\left|H_{b}(f)\right|_{\mathbb{R}^{m}}^{2} .
$$

Moreover, the equality holds in this inequality if and only if $f(M)$ is contained in a sphere $\mathbb{S}^{m-1}(r)$ of radius $r=\sqrt{\frac{2 n}{\lambda_{2}\left(-\Delta_{b}\right)}}$ and $f$ is a pseudoharmonic map from $(M, \theta)$ to the sphere $S^{m-1}(r)$.

Similarly, for CR manifolds mapped into the Heisenberg group, one has the following

Theorem 6.2. Let $(M, \theta)$ be a compact strictly pseudoconvex $C R$ manifold of dimension $2 n+1$ without boundary.

i) Let $f: M \longrightarrow \mathbb{H}^{m}=\mathbb{R}^{2 m} \times \mathbb{R}$ be any $C^{2}$ map satisfying $d f(H(M)) \subseteq$ $H\left(\mathbb{H}^{m}\right)$. Then

$$
\lambda_{2}\left(-\Delta_{b}\right) E_{b}(f) \leq \frac{1}{2} \int_{M}\left|H_{b}(f)\right|_{\mathbb{H}^{m}}^{2}
$$

where the equality holds if and only if the first $2 m$ components $f_{1}, \ldots, f_{2 m}$ of $f$ satisfy $-\Delta_{b} f_{\alpha}=\lambda_{2}\left(-\Delta_{b}\right)\left(f_{\alpha}-f f_{\alpha}\right)$ for every $\alpha \leq 2 \mathrm{~m}$.

ii) Let $f: M \longrightarrow \mathbb{H}^{m}$ be any $C^{2}$ semi-isometric map satisfying $d f(H(M)) \subseteq$ $H\left(\mathbb{H}^{m}\right)$. Then

$$
\lambda_{2}\left(-\Delta_{b}\right) \leq \frac{1}{2 n V(M, \theta)} \int_{M}\left|H_{b}(f)\right|_{\mathbb{H}^{m}}^{2} .
$$

Moreover, the equality holds in this last inequality if and only if $f(M)$ is contained in the product $\mathbb{S}^{2 m-1}(r) \times \mathbb{R} \subset \mathbb{H}^{m}$ with $r=\sqrt{\frac{2 n}{\lambda_{2}\left(-\Delta_{b}\right)}}$, and $\pi \circ f$ is a pseudo-harmonic map from $(M, \theta)$ to the sphere $S^{2 m-1}(r)$, where $\pi: \mathbb{H}^{m} \rightarrow$ $\mathbb{R}^{2 m}$ is the standard projection.

Proof. i) Let $f: M \longrightarrow \mathbb{H}^{m}=\mathbb{R}^{2 m} \times \mathbb{R}$ be a $C^{2}$ map satisfying $d f(H(M)) \subseteq$ $H\left(\mathbb{H}^{m}\right)$ and set $\tilde{f}:=\pi \circ f: M \longrightarrow \mathbb{R}^{2 m}$ where $\pi: \mathbb{H}^{m} \rightarrow \mathbb{R}^{2 m}$ is the standard projection. One has, for every pair $(X, Y)$ of horizontal vectors,

$$
\beta_{\tilde{f}}(X, Y)=\beta_{\pi}(d f(X), d f(Y))+d \pi\left(\beta_{f}(X, Y)\right) .
$$

Since for any $X \in H\left(\mathbb{H}^{m}\right),|d \pi(X)|_{\mathbb{R}^{2 m}}^{2}=\frac{1}{4}|X|_{\mathbb{H}^{m}}^{2}$ (see $(\underline{5.3)})$ and $d \pi(T)=0$, one can easily check that $\beta_{\pi} \equiv 0$ (Corollary 2.1) and, then, $\beta_{\tilde{f}}(X, Y)=$ $d \pi\left(\beta_{f}(X, Y)\right)$. Thus, $H_{b}(\tilde{f})=d \pi\left(H_{b}(f)\right)$ and, since $H_{b}(f)$ is horizontal 
(Proposition 5.1), $\left|H_{b}(\tilde{f})\right|_{\mathbb{R}^{2 m}}^{2}=\frac{1}{4}\left|H_{b}(f)\right|_{\mathbb{H}^{m}}^{2}$. On the other hand, it is clear that $e_{b}(\tilde{f})=\frac{1}{4} e_{b}(f)$ and, then, $E_{b}(\tilde{f})=\frac{1}{4} E_{b}(f)$. Therefore, it suffices to apply Theorem 6.1 to complete the proof of the first part of the theorem.

ii) Assume now that the map $f$ is semi-isometric. Using the assumption that $f$ preserves horizontality, i.e., $d f(H(M)) \subseteq H\left(\mathbb{H}^{m}\right)$, one checks that the map $2 \pi \circ f$ is also semi-isometric. Applying Corollary 6.1 to the latter we easily deduce what is stated in part (ii) of the theorem.

\section{Eigenvalues of the Horizontal Laplacian on a Carnot group}

A Carnot group of step $r$ is a connected, simply connected, nilpotent Lie group $G$ whose Lie algebra $\mathfrak{g}$ admits a stratification

$$
\mathfrak{g}=V_{1} \oplus \ldots \oplus V_{r}
$$

so that $\left[V_{1}, V_{j}\right]=V_{j+1}, j=1, \ldots, r-1$ and $\left[V_{i}, V_{j}\right] \subset V_{i+j}, j=1, \ldots, r$, with $V_{k}=\{0\}$ for $k>r$. We also assume that $\mathfrak{g}$ carries a scalar product $\langle,\rangle_{\mathfrak{g}}$ for which the subspaces $V_{j}$ are mutually orthogonal. The layer $V_{1}$ generates the whole $g$ and induces a sub-bundle $H G$ of $T G$ of rank $d_{1}=\operatorname{dim} V_{1}$ that we call the horizontal bundle of the Carnot group. The Heisenberg group $\mathbb{H}^{d}$ is the simplest example of a Carnot group of step 2.

For each $i \leq r$, let $\left\{e_{1}^{i}, \cdots, e_{d_{i}}^{i}\right\}$ be an orthonormal basis of $V_{i}$ and denote by $\left\{X_{1}^{i}, \cdots, X_{d_{i}}^{i}\right\}$ the system of left invariant vector fields that coincides with $\left\{e_{1}^{i}, \cdots, e_{d_{i}}^{i}\right\}$ at the identity element of $G$. We consider the Riemannian metric $g_{G}$ on $G$ with respect to which the family $\left\{X_{1}^{1}, \cdots, X_{d_{1}}^{1}, \cdots, X_{1}^{r}, \cdots, X_{d_{r}}^{r}\right\}$ constitute an orthonormal frame for $T G$. The corresponding Levi-Civita connection $\nabla$ induces a connection $\nabla^{H}$ on $H G$ that we call "horizontal connection" : If $\mathrm{X}$ and $Y$ are smooth sections of $H G$, then $\nabla_{X}^{H} Y=\pi_{H} \nabla_{X} Y$, where $\pi_{H}: T G \rightarrow H G$ is the orthogonal projection. The horizontal Laplacian $\Delta_{H}$ is then defined for every $C^{2}$ function on $G$ by

$$
\Delta_{H} u:=\operatorname{trace}_{H} \nabla^{H} d u=\sum_{i \leq d_{1}} X_{i}^{1} \cdot\left(X_{i}^{1} \cdot u\right),
$$

where the last equality follows from the fact that $\nabla_{X_{1}^{i}}^{H} X_{1}^{j}=0$ for any $i, j=$ $1 \ldots d_{1}$. The operator $\Delta_{H}$ is a hypoelliptic operator of Hörmander type.

Theorem 7.1. Let $G$ be a Carnot group and let $\Omega$ be a bounded domain in $G$. Let $V$ be a function on $\Omega$ so that the operator $-\Delta_{H}+V$, with Dirichlet boundary conditions if $\Omega \neq G$, admits a purely discrete spectrum $\left\{\lambda_{j}\right\}_{j \geq 1}$ which is bounded from below. Then, for every $k \geq 1$ and $p \in \mathbb{R}$,

$$
\sum_{i=1}^{k}\left(\lambda_{k+1}-\lambda_{i}\right)^{p} \leq \frac{\max \{4,2 p\}}{d} \sum_{i=1}^{k}\left(\lambda_{k+1}-\lambda_{i}\right)^{p-1}\left(\lambda_{i}-T_{i}\right),
$$


where $d$ is the rank of the horizontal distribution $H G, T_{i}=\int_{\Omega} V u_{i}^{2} v_{G}$ and $v_{G}$ is the Riemannian volume element associated with $g_{G}$. Moreover, if $V$ is bounded below on $\Omega$, then for every $k \geq 1$,

$$
\lambda_{k+1} \leq\left(1+\frac{4}{d}\right) \frac{1}{k} \sum_{i=1}^{k} \lambda_{i}-\frac{4}{d} \inf _{\Omega} V
$$

and

$$
\lambda_{k+1} \leq\left(1+\frac{4}{d}\right) k^{\frac{2}{d}} \lambda_{1}-C(d, k) \inf _{\Omega} V
$$

with $C(d, k)=\left(1+\frac{4}{d}\right) k^{\frac{2}{d}}-1$.

Proof. Let $\left\{e_{1}, \ldots, e_{d}\right\}$ be an orthonormal basis of the subspace $V_{1}$ and denote by $\left\{X_{1}, \cdots, X_{d}\right\}$ the system of left invariant vector fields that coincides with $\left\{e_{1}, \ldots, e_{d}\right\}$ at the identity element of $G$. Since the group $G$ is nilpotent, the exponential map exp $: \mathfrak{g} \longrightarrow G$ is a global diffeomorphism. We can define, for each $i \leq d$, a smooth map $x_{i}: G \rightarrow \mathbb{R}$ by

$$
x_{i}(g):=\left\langle\exp ^{-1}(g), e_{i}\right\rangle_{\mathrm{g}} .
$$

These functions satisfy (see [12, Proposition 5.7]), $\forall i, j=1, \ldots, m$,

$$
X_{j} \cdot x_{i}=\delta_{i j} \text { and } \Delta_{H} x_{i}=0 .
$$

Again, we apply Lemma 3.1 with $A=-\Delta_{H}+V$ and $B=x_{\alpha}, 1 \leq \alpha \leq$ $m$. We need to deal with the calculation of $\left\langle\left[-\Delta_{H}+V, x_{\alpha}\right] u_{i}, x_{\alpha} u_{i}\right\rangle_{L^{2}}$ and $\left\|\left[-\Delta_{H}+V, x_{\alpha}\right] u_{i}\right\|_{L^{2}}^{2}$, where $\left\{u_{i}\right\}_{i \geq 1}$ a complete orthonormal family of eigenfunctions with $\left(-\Delta_{b}+V\right) u_{i}=\lambda_{i} u_{i}$. We have after a straightforward calculation :

$$
\left[-\Delta_{H}+V, x_{\alpha}\right] u_{i}=-2 X_{\alpha} \cdot u_{i}
$$

Integrating by parts we get

$$
\int_{\Omega}\left(X_{\alpha} \cdot u_{i}\right) x_{\alpha} u_{i}=\frac{1}{2} \int_{\Omega}\left(X_{\alpha} \cdot u_{i}^{2}\right) x_{\alpha}=-\frac{1}{2} \int_{\Omega} u_{i}^{2}\left(X_{\alpha} \cdot x_{\alpha}\right)=-\frac{1}{2} \int_{\Omega} u_{i}^{2}=-\frac{1}{2} .
$$

Thus,

$$
\sum_{\alpha=1}^{d}\left\langle\left[-\Delta_{H}+V, x_{\alpha}\right] u_{i}, x_{\alpha} u_{i}\right\rangle_{L^{2}}=-2 \sum_{\alpha=1}^{d} \int_{\Omega}\left(X_{\alpha} \cdot u_{i}\right) x_{\alpha} u_{i}=d .
$$

On the other hand, we have

$$
\sum_{\alpha=1}^{d}\left\|\left[-\Delta_{H}+V, x_{\alpha}\right] u_{i}\right\|_{L^{2}}^{2}=4 \sum_{\alpha=1}^{d} \int_{\Omega}\left|X_{\alpha} \cdot u_{i}\right|^{2}=4\left(\lambda_{i}-T_{i}\right)
$$

Putting these identities in Lemma 3.1, we obtain the first inequality of the theorem.

The rest of the proof is identical to that of Theorem 3.1 . 


\section{REFERENCES}

[1] Mark S. Ashbaugh. The universal eigenvalue bounds of Payne-Pólya-Weinberger, Hile-Protter, and H. C. Yang. Proc. Indian Acad. Sci. Math. Sci., 112(1):3-30, 2002. Spectral and inverse spectral theory (Goa, 2000).

[2] M.S. Ashbaugh and L. Hermi. On Harrell-Stubbe type inequalities for the discrete spectrum of a self-adjoint operator. arXiv:0712.4396v1 [math.SP], page 42 pages, 2007.

[3] E. Barletta and S. Dragomir. On the spectrum of a strictly pseudoconvex CR manifold. Abh. Math. Sem. Univ. Hamburg, 67:33-46, 1997.

[4] Elisabetta Barletta. The Lichnerowicz theorem on CR manifolds. Tsukuba J. Math., 31(1):77-97, 2007.

[5] Elisabetta Barletta and Sorin Dragomir. Sublaplacians on CR manifolds. Bull. Math. Soc. Sci. Math. Roumanie (N.S.), 52(100)(1):3-32, 2009.

[6] Elisabetta Barletta, Sorin Dragomir, and Hajime Urakawa. Pseudoharmonic maps from nondegenerate CR manifolds to Riemannian manifolds. Indiana Univ. Math. J., 50(2):719-746, 2001.

[7] Elisabetta Barletta, Sorin Dragomir, and Hajime Urakawa. Yang-Mills fields on CR manifolds. J. Math. Phys., 47(8):083504, 41, 2006.

[8] Shu-Cheng Chang and Hung-Lin Chiu. On the CR analogue of Obata's theorem in a pseudohermitian 3-manifold. Math. Ann., 345(1):33-51, 2009.

[9] Daguang Chen and Qing-Ming Cheng. Extrinsic estimates for eigenvalues of the Laplace operator. J. Math. Soc. Japan, 60(2):325-339, 2008.

[10] Qing-Ming Cheng and Hongcang Yang. Estimates on eigenvalues of Laplacian. Math. Ann., 331(2):445-460, 2005.

[11] Qing-Ming Cheng and Hongcang Yang. Bounds on eigenvalues of Dirichlet Laplacian. Math. Ann., 337(1):159-175, 2007.

[12] D. Danielli, N. Garofalo, and D. M. Nhieu. Sub-Riemannian calculus on hypersurfaces in Carnot groups. Adv. Math., 215(1):292-378, 2007.

[13] Sorin Dragomir and Giuseppe Tomassini. Differential geometry and analysis on CR manifolds, volume 246 of Progress in Mathematics. Birkhäuser Boston Inc., Boston, MA, 2006.

[14] J. Eells and L. Lemaire. Another report on harmonic maps. Bull. London Math. Soc., 20(5):385-524, 1988.

[15] A. El Soufi and S. Ilias. Une inégalité du type "Reilly" pour les sous-variétés de l'espace hyperbolique. Comment. Math. Helv., 67(2):167-181, 1992.

[16] Ahmad El Soufi, Evans M. Harrell, II, and Saïd Ilias. Universal inequalities for the eigenvalues of Laplace and Schrödinger operators on submanifolds. Trans. Amer. Math. Soc., 361(5):2337-2350, 2009.

[17] Allan Greenleaf. The first eigenvalue of a sub-Laplacian on a pseudo-Hermitian manifold. Comm. Partial Differential Equations, 10(2):191-217, 1985.

[18] Evans M. Harrell, II. Some geometric bounds on eigenvalue gaps. Comm. Partial Differential Equations, 18(1-2):179-198, 1993.

[19] Evans M. Harrell, II. Commutators, eigenvalue gaps, and mean curvature in the theory of Schrödinger operators. Comm. Partial Differential Equations, 32(1-3):401-413, 2007.

[20] Evans M. Harrell, II and Patricia L. Michel. Commutator bounds for eigenvalues, with applications to spectral geometry. Comm. Partial Differential Equations, 19(1112):2037-2055, 1994.

[21] Evans M. Harrell, II and Joachim Stubbe. On trace identities and universal eigenvalue estimates for some partial differential operators. Trans. Amer. Math. Soc., 349(5):1797-1809, 1997. 
[22] Evans M. Harrell, II and Joachim Stubbe. Universal bounds and semiclassical estimates for eigenvalues of abstract Schrödinger operators. SIAM J. Math. Anal., 42(5):2261-2274, 2010.

[23] G. N. Hile and M. H. Protter. Inequalities for eigenvalues of the Laplacian. Indiana Univ. Math. J., 29(4):523-538, 1980.

[24] Song-Ying Li and Hing-Sun Luk. The sharp lower bound for the first positive eigenvalue of a sub-Laplacian on a pseudo-Hermitian manifold. Proc. Amer. Math. Soc., 132(3):789-798 (electronic), 2004.

[25] A. Menikoff and J. Sjöstrand. On the eigenvalues of a class of hypoelliptic operators. Math. Ann., 235(1):55-85, 1978.

[26] Pengcheng Niu and Huiqing Zhang. Payne-Polya-Weinberger type inequalities for eigenvalues of nonelliptic operators. Pacific J. Math., 208(2):325-345, 2003.

[27] L. E. Payne, G. Pólya, and H. F. Weinberger. On the ratio of consecutive eigenvalues. J. Math. and Phys., 35:289-298, 1956.

[28] Xiao Chun Peng and Wen Yi Chen. Spectra of subelliptic operators on $S^{3}$. J. Math. (Wuhan), 29(3):297-299, 2009.

[29] Raphaël S. Ponge. Heisenberg calculus and spectral theory of hypoelliptic operators on Heisenberg manifolds. Mem. Amer. Math. Soc., 194(906):viii+ 134, 2008.

[30] H. C. Yang. An estimate of the difference between consecutive eigenvalues. preprint IC/91/60 of the Intl., Revised version, preprint 1995.

Amine Aribi : Laboratoire de Mathématiques et Physique Théorique, UMR-CNRS 6083, Université François Rabelais de Tours, Parc de Grandmont, 37200 Tours, France E-mail address: Amine.Aribi@lmpt.univ-tours. fr

Laboratoire de Mathématiques et Physique Théorique, UMR-CNRS 6083, Université François Rabelais de Tours, Parc de Grandmont, 37200 Tours, France

E-mail address: Ahmad.Elsoufi@lmpt.univ-tours.fr 